\title{
Long non-coding RNA NEAT1-modulated abnormal lipolysis via ATGL drives hepatocellular carcinoma proliferation
}

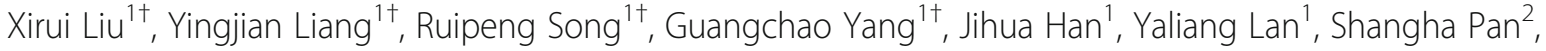 \\ Mingxi Zhu', Yao Liu', Yan Wang ${ }^{1}$, Fanzheng Meng ${ }^{1}$, Yifeng Cui ${ }^{1}$, Jiabei Wang ${ }^{1}$, Bo Zhang ${ }^{1}$, Xuan Song ${ }^{1}$, \\ Zhaoyang Lu', Tongsen Zheng ${ }^{3}$ and Lianxin Liu' ${ }^{1,4^{*}}$
}

\begin{abstract}
Background: Abnormal metabolism, including abnormal lipid metabolism, is a hallmark of cancer cells. Some studies have demonstrated that the lipogenic pathway might promote the development of hepatocellular carcinoma (HCC). However, the role of the lipolytic pathway in HCC has not been elucidated.
\end{abstract}

Methods: We compared levels of adipose triglyceride lipase (ATGL) in human HCC and healthy liver tissues by real time PCR, western blot and immunohistochemistry. We measured diacylglycerol(DAG) and free fatty acid (FFA) levels in HCC cells driven by the NEAT1-ATGL axis and in HCC tissues. We also assessed the effects of ATGL, DAG, FFA, and NEAT1 on HCC cells proliferation in vitro and in an orthotopic xenograft HCC mouse model. We also performed a luciferase reporter assay to investigate the interaction between NEAT1/ATGL and miR-124-3p.

Results: We found that the lipolytic enzyme, ATGL is highly expressed in human HCC tissues and predicts poor prognosis. We also found that high levels of DAG and FFA are present in HCC tissues. Furthermore, the IncRNANEAT1 was found to modulate ATGL expression and disrupt lipolysis in HCC cells via ATGL. Notably, ATGL and its products, DAG and FFA, were shown to be responsible for NEAT1-mediated HCC cell growth. NEAT1 regulated ATGL expression by binding miR-124-3p. Additionally, NEAT1 knockdown attenuated HCC cell growth through miR-1243P/ATGL/DAG+FFA/PPARa signaling.

Conclusion: Our results reveal that NEAT1-modulates abnormal lipolysis via ATGL to drive HCC proliferation.

Keywords: Hepatocellular carcinoma, NEAT1, ATGL/PNPLA2, Lipolysis, LncRNA, Lipid metablosim

\section{Background}

Hepatocellular carcinoma (HCC) is the sixth most common cancer worldwide and the second leading cause of cancer death in men [1]. The progression of HCC is characterized by abnormal cell differentiation, fast infiltrating growth, early metastasis, high-grade malignancy, and poor prognosis [2]. Abnormal metabolism, including abnormal

\footnotetext{
* Correspondence: LiuLX@ems.hrbmu.edu.cn

${ }^{\dagger}$ Equal contributors

'Department of Hepatic Surgery, The First Affiliated Hospital of Harbin Medical University, Key Laboratory of Hepatosplenic Surgery, Ministry of Education, Harbin, Heilongjiang Province, China

${ }^{4}$ Department of Pharmacology (the State-Province Key Laboratories of Biomedicine-Pharmaceutics of China, Key Laboratory of Cardiovascular Research, Ministry of Education), Harbin Medical University, Harbin, China Full list of author information is available at the end of the article
}

lipid metabolism, is a hallmark of cancer cells. Large amounts of FAs are required to accommodate high rates of proliferation in cancer cells [3]. Cancer cells can acquire FAs through lipogenic and lipolytic pathways [4].

Currently, it is believe that adipose triglyceride lipase (ATGL), hormone-sensitive lipase (HSL) and monoacyglycerol lipase (MAGL) are the main enzymes involved in lipolysis [5]. ATGL initiates the process of triglyceride (TAG) metabolism by hydrolyzing TAG into diacylglycerol (DAG) and FFA. The subsequent step requires HSL, which breaks down DAG into monoacylglycerol (MAG). Finally, MAG is further broken down into FFA and glycerol by MAGL. Daniel et al. revealed that the lipolytic enzyme, MAGL promotes migration, survival, and in vivo tumor growth through the MAGL- free fatty acid (FFA) pathway [6].

(c) The Author(s). 2018 Open Access This article is distributed under the terms of the Creative Commons Attribution 4.0 International License (http://creativecommons.org/licenses/by/4.0/), which permits unrestricted use, distribution, and 
However, other studies indicated that MAGL acts as a tumor suppressor role [7]. These studies indicate that the role of lipolytic enzymes in tumor progression requires further study. Although, recent studies have revealed that the inhibition of ATGL via RNAi or a small molecule inhibitor attenuated the growth and motility of tumor cells (colorectal cancer cells and non-small cell lung carcinomas cells) [8], However, whether it contributes to tumor growth, or other functions in HCC remains unclear.

Nuclear paraspeckle assembly transcript 1 (NEAT1) is a nuclear-enriched lncRNA and is a scaffolding factor that is necessary for the formation of nuclear paraspeckles [9]. NEAT1 is up-regulated in various types of cancers and has been reported to be associated with unfavorable prognosis in cancer patients [10]. NEAT1 was demonstrated to function as a competing endogenous RNA (ceRNA) by competitively binding common microRNAs [11, 12]. Although recent studies have demonstrated that NEAT1 is overexpressed specifically in HCC [13], the mechanism through which NEAT1 affects tumor progression requires further study.

We hereby report that the lncRNA-NEAT1 disrupts HCC cell lipolysis through ATGL. Our results explain the high levels of DAG and FFA present in HCC tissues. ATGL and its products, DAG and FFA, are responsible for NEAT1-mediated HCC cell growth. Additionally, NEAT1 mediates HCC cell growth through the miR-124$3 \mathrm{p} / \mathrm{ATGL} / \mathrm{DAG}+\mathrm{FFA} / \mathrm{PPAR} \alpha$ pathway. Thus, we demonstrate that NEAT1-mediated abnormal lipolysis promotes $\mathrm{HCC}$ cell growth.

\section{Methods}

Patients and tissue samples

From 2008 to 2012, archival HCC tissues were obtained from patients at the First Affiliated Hospital of Harbin Medical University. Informed consent was obtained from each patient prior to biopsy or surgery, and ethical approval for the use of human subjects was obtained from the Research Ethics Committee of the First Affiliated Hospital of Harbin Medical University. Detailed characteristics of patients were summarized in Additional file 1: Table S1 and Table S2.

\section{Cell lines and culture conditions}

The L02 immortalized liver cell line and $293 \mathrm{~T}$ was purchased from the Institute of Biochemistry and Cell Biology, Chinese Academy of Science, China. HepG2, Huh7, SKHep-1, and HCCLM3 were purchased from the American Type Culture Collection (Manassas, VA, USA). Huh7luciferase-transfected and HCCLM3-luciferase-transfected cells were purchased from Berthold Technologies. All cell lines were cultured in DMEM supplemented with $10 \%$ FBS, 100 units $/ \mathrm{mL}$ penicillin, and $100 \mu \mathrm{g} / \mathrm{mL}$ streptomycin.

\section{Lentiviral infection}

Human Lenti-shNEAT1-GFP, Lenti-shPNPLA2-GFP, Lenti-PNPLA2-GFP, Lenti-vector-GFP control and Lenti-sh-control-GFP were designed and purchased from GeneChem Technologies (Shanghai, China). Transfection was performed according to standard procedures. The shRNA sequences used in this study are listed as follow:

\section{NEAT1-shRNA\#1:GTGAGAAGTTGCTTAGAAA; NEAT1-shRNA\#2:TGGTAATGGTGGAGGAAGA; ATGL(PNPLA2)-shRNA\#1:AAGTTCATTGAGGT ATCTA;ATGL(PNPLA2)-shRNA\#2:CTTTACTCCT GAGAACTTT.}

\section{Transient transfection}

Small interfering RNA (siRNA), si-control, miR-124-3p mimic, miR-124-3p miR-124-3p inhibitor, negative control were purchased from Ribobio (Guangzhou, China). For transfection, miR-124-3p mimic, miR-124-3p inhibitor, negative control, siRNA or si-control in Lipofectamine 2000 (Invitrogen) was transfected into cells according to the manufacturer's instructions. The siRNA sequences are listed in Additional file 2: Table S3.

\section{Cell proliferation assay}

For the Cell Counting Kit-8 assay (CCK-8), cells were seeded at a density of 2500-4000 cells/well in 96-well plates. In vitro cell proliferation was assessed by Cell Counting Kit-8 (Dojindo) according to the manufacturer's instructions. For the cell growth assays, HCC cells were seeded at a density of $0.5 \times 10^{4}$ per well. The number of viable cells was determined at different timepoints. For the Colony formation assay, cells were seeded in 6-well plates at a density of 500-800 cells/well and cultured for 14 days. Colonies were stained with $0.5 \%$ Crystal Violet for $10 \mathrm{~min}$ and counted.

\section{Measurement of DAG and FFA contents}

DAG and FFA contents were determined with a DAG ELISA Kit (BlueGene Biotech, E01D0010) and Free Fatty Acid Quantitation Kit (Sigma, MAK044) respectively, following the manufacturers' protocols.

\section{Western blot}

Western blot analysis was performed as previously described [14]. In brief, whole cell or tissue extracts were prepared using RIPA buffer. After electrophoresis, proteins were electroeluted at 120 Volts onto a polyvinylidenedifluoride (PVDF) membrane (Invitrogen). Indicated primary antibodies were used. Protein bands were visualized by an enhanced chemiluminescence assay kit (Super Signal Pierce Bio-technology). The following antibodies against ATGL/PNPLA2(Cayman, 10006409), 
PPAR $\alpha($ Abcam, ab8934), MAGL(Abcam, ab24701), HSL(Abcam,ab45422), p53(Santa Cruz, SC126) and p21(Abcam, ab109520), Bax (Abcam, ab32503), ß-Actin (Cell Signaling Technology) were used. Western blot analyses were repeated at least three times.

\section{Immunohistochemical analysis}

Expression of Ki-67 and ATGL was evaluated using an immunohistochemical (IHC) method described previously [14].

\section{Reagents}

1,3-Dilinoleoyl-rac-glycerol (Sigma, D9508) was dissolved in fresh dimethyl sulfoxide (DMSO) for stock solution at $50 \mathrm{mM}$ (or $50 \mathrm{mg} / \mathrm{ml}$ for the in vivo study). Similarly, oleic acid (Sigma, O1008) was dissolved in fresh DMSO to $50 \mathrm{mM}$ (or $50 \mathrm{mg} / \mathrm{ml}$ for the in vivo study). 1,3-Dilinoleoyl-rac-glycerol and oleic acid were mixed at a 1:1 ratio to prepare the DAG+FFA mixture. Working solution was added with pre-set DAG and FFA concentrations by mixing common serum-free medium proportionately. Final concentrations were set at $8 \mu \mathrm{M}$, $16 \mu \mathrm{M}$ and $32 \mu \mathrm{M}$. Atglistatin (Sigma, SML1075) was dissolved in DMSO for stock solution at $10 \mathrm{mM}$. Final working concentrations of $40 \mu \mathrm{M}$ Atglistatin was used in all experiments. Nutlin-3a(Sigma, SML0580) was dissolved in DMSO for stock solution at $5 \mathrm{mg} / \mathrm{ml}$. Final working concentrations of $10 \mu \mathrm{M}$ Nutlin-3a was used in experiments.

\section{Real time PCR analysis}

Total RNA was extracted from cultured cells using the RNAeasy Mini kit (Qiagen, Valencia, CA, USA) according to the manufacturer's instructions. Reverse transcription was performed using the High Capacity Reverse Transcription kit (Applied Biosystems, Foster City, CA, USA) after RNA quantification. Real-time PCR was performed using the Power SYBR Green PCR Master Mix (Life Technologies, Carlsbad, CA, USA) on an ABI Prism 7900HT instrument (Applied Biosystems). Realtime PCR was performed in triplicate. The expression of lncRNA and protein coding genes were normalized to that of $\beta$-Actin gene. Specifically, stem-loop reverse transcriptase polymerase chain reaction was used in the analysis of mature miRNA expression. The stem-loop RT primer was CCTGTTGTCTCCAGCCACAAAAGAGCA CAATATTTCAGGAGACAACAGGGGCATTC. The reverse transcriptase reactions was performed as described previously [15]. Reverse transcriptase reactions contained $2 \mu \mathrm{g}$ total RNA, $50 \mathrm{nM}$ stem-loop RT primer, 1× RT buffer (Applied Biosystems, Foster City, CA, USA), $0.25 \mathrm{mM}$ dNTPs, $3.33 \mathrm{U} / \mu \mathrm{l}$ MultiScribe reverse transcriptase (Applied Biosystems, Foster City, CA, USA) and $0.25 \mathrm{U} / \mu \mathrm{l}$ RNase inhibitor (Applied Biosystems, Foster
City, CA, USA). The reaction parameters were as follows: $30 \mathrm{~min}$ at $16^{\circ} \mathrm{C}, 30 \mathrm{~min}$ at $42{ }^{\circ} \mathrm{C}, 5 \mathrm{~min}$ at $85^{\circ} \mathrm{C}$ and then held at $4{ }^{\circ} \mathrm{C}$. Quantitative RT-PCR reaction parameters were as follows: $2 \mathrm{~min}$ at $50{ }^{\circ} \mathrm{C}, 2 \mathrm{~min}$ at $95{ }^{\circ} \mathrm{C}, 40$ cycles of denaturation at $95{ }^{\circ} \mathrm{C}$ for $30 \mathrm{~s}$, Annealing at $60{ }^{\circ} \mathrm{C}$ for $1 \mathrm{~min}$. The expression of miRNA was normalized to that of U6 gene. Expression levels of target genes were determined according to the $2^{-\Delta \Delta \mathrm{Ct}}$ method. Primers used are listed in Additional file 2: Table S4.

\section{Fluorescent In Situ Hybridization (FISH)}

Fluorescent In situ hybridization (FISH) was performed with a Fluorescent In Situ Hybridization Kit (RiboBio, Guangzhou, China), following the manufacturers' protocols. The CY3 labeled miR-124-3p probe were designed and purchased from GenePharma Technologies (Shanghai, China). The sequence was: 5'-GGCAUUCACCGCGUGCCUUA-3'.

\section{P53 mutational analysis by PCR and direct sequencing}

The mutation detection of the p53 gene was carried out by amplification of exons 2-11 from genomic DNA with 7 pairs of primers. Primer sequences are listed in Additional file 2: Table S5. Ex TaqDNA Polymerase used in PCR amplification was purchased from Takara, Japan. Amplification was done following the manufacturers' protocols. PCR products were sequenced using an ABI3730XL DNA sequencer (Applied Biosystems). Profiles of p53 mutation were showed in Additional file 2: Table S6.

\section{Animal studies}

Male BALB/c nude mice (4-6 weeks old) were obtained from the experimental animal center of the Shanghai Institute for Biological Sciences (SIBS) and housed under standard conditions and care according to the institutional guidelines for animal care. All animal experiments were approved by the Institutional Animal Care and Use Committee of Harbin Medical University. To establish an orthotopic HCC mouse model, $4 \times 10^{6}$ cells in $100 \mu \mathrm{L}$ of phosphate-buffered saline were subcutaneously injected into the flanks of nude mice. After 1-2 weeks, the subcutaneous tumors were resected and diced into $1 \mathrm{~mm}^{3}$ cubes, which were then implanted into the left lobes of the livers of the nude mice. A DAG and FFA mixture was used in vivo experiment. DAG and FFA solution was prepared in $20 \%$ DMSO and $15 \%$ Tween 80 in $0.9 \%$ saline. After 1 week of implantation mice were injected $20 \mathrm{mg} / \mathrm{kg}$ DAG+FFA intraperitoneally for 5 weeks ( 5 days per week). Mice were imaged by the bioluminescence IVIS Imaging System weekly and mice were then sacrificed. 


\section{Luciferase reporter assay}

The luciferase reporter assay was performed according to the method described previously [16].

\section{Statistical analysis}

Statistical analysis was performed with the GraphPad Prism software package (v. 4.02; San Diego, CA, USA) or SPSS 16.0 software (Chicago, IL, USA). Student's t-test or one-way ANOVA was applied to determine the significance between groups. Statistical analyses between different treatments, in different cell cohorts or at different time points were performed using two-way ANOVA with the Bonferroni's correction. Overall survival (OS) was compared with the KaplanMeier method, and the significance was determined by the log-rank test. Correlations were calculated using Spearman rank-order coefficients. Values were expressed as mean $\pm \mathrm{SD}$ values. Statistically significant was concluded at ${ }^{*} P<0.05,{ }^{* *} P<0.01$, ${ }^{* * * *} P<0.001$; NS represents no statistically significant.

\section{Results \\ ATGL is highly expressed in human HCC tissues and predicts a poor prognosis}

The intracellular lipolytic pathway is currently known to be mainly regulated by three enzymes: namely, ATGL, HSL and MAGL [5]. Thus, we first elucidated whether these three enzymes were aberrantly expressed in five pairs of HCC tissues and adjacent non-tumorous liver tissues. The results indicated that ATGL mRNA was more aberrantly expressed in HCC tissues than HSL and MAGL mRNA (Additional file 3: Figure S1A). Next, we evaluated ATGL expression in 40 pairs of HCC tissues. Quantitative real-time PCR (qRT-PCR) revealed that mRNA levels of ATGL were higher in HCC tissues than in their adjacent non-tumorous liver tissues (Fig. 1a). Moreover, we found that ATGL expression was positively correlated with tumor size. However, it was not correlated with HBV infection (Additional file 1: Table S1). We also evaluated the expression of ATGL by western blot in clinical HCC tissues. The expression of ATGL was higher in $\mathrm{HCC}$ tissues than in their adjacent non-tumorous liver tissues (Fig. 1b). In addition, we evaluated the expression of ATGL by immunohistochemical (IHC) staining in clinical HCC tissues using tissue microarrays and found that 76 . $11 \%(86 / 113)$ were positive in HCC tissues compared with $12.20 \%(5 / 41)$ in normal liver tissues (Fig. 1c). Moreover, ATGL expression was negatively correlated with patient survival in 40 HCC tissues (Fig. 1d). To elucidate the effect of ATGL on HCC carcinogenesis, we analyzed ATGL protein and mRNA levels in a panel of HCC cell lines. The results indicated that ATGL protein and mRNA levels increased progressively from healthy liver cells to HCC cells with low growth potential and, finally, to HCC cells with high growth potential (Fig. 1e, f).

ATGL has been reported to initiate the process of TAG metabolism by hydrolyzing TAG into DAG and FFA [17]. To confirm this process in HCC cells, we generated lentiviral constructs expressing ATGL-GFP and sh-ATGL-GFP to infect HCC cells. Western blot results confirmed remarkable ATGL overexpression (Additional file 3: Figure S1B). Simultaneously, Compared with the control shRNA, ATGL expression was notably decreased by lenti-sh-ATGL\#2 as shown in Additional file 3: Figure S1B. Thus, sh-ATGL-\#2 was chosen for further experiments. Our data indicated that overexpression of ATGL increased intracellular FFA and DAG levels in HCC cells (Additional file 3: Figure S1C). Similarly, ATGL knockdown or treatment with Atglistatin (selective inhibitor of ATGL) reduced in intracellular FFA and DAG levels (Additional file 3: Figure S1D). In addition, we found that higher levels of DAG and FFA accumulated in HCC tissues compared with in the corresponding peritumoral tissues (Fig. 1g). These experimental results suggested that ATGL was highly expressed and maintained elevated levels of DAG and FFA in human HCC tissues. This indicated that ATGL and its products, DAG and FFA, may play important roles in HCC development.

\section{ATGL promotes HCC cell growth by maintaining elevated} levels of DAG and FFA in vitro and in vivo

We next assess the effects of ATGL on the growth of HCC cells. According to growth curves and CCK-8 assay results, the up-regulation of ATGL significantly promoted Huh7 and HepG2 cell growth (FIig.2a and Additional file 4: Figure S2A). Colony formation assay also revealed that up-regulating ATGL expression resulted in more visible colonies compared with control in Huh7 and HepG2 cell lines (Fig. 2b and Additional file 4: Figure S2B and C).

Next we explored whether DAG and FFA facilitates HCC cell growth. CCK- 8 assay results showed that treatment with DAG and FFA separately facilitated HCC cell growth in a dose-dependent manner (Additional file 5: Figure S3A and B). Simultaneously, treatment with $16 \mu \mathrm{M}$ DAG together with $16 \mu \mathrm{M}$ FFA $(16 \mu \mathrm{M}$ DAG+ FFA) in HCC cells resulted in a synergistic effect to facilitate HCC cell growth (Additional file 5: Figure S3C).

Considering the important roles of DAG and FFA in the promotion of HCC cell growth, we hypothesized that the pro-tumorigenic effects of ATGL were mediated by DAG+FFA. We reasoned that, if the pro-tumorigenic effects of ATGL were mediated by DAG+FFA, then the impaired pathogenicity of sh-ATGL cancer cells might be rescued by treatment with exogenous sources of DAG+FFA. To test this hypothesis, we treated sh-ATGL HCC cells with $16 \mu \mathrm{M}$ DAG+FFA. According to growth 
a

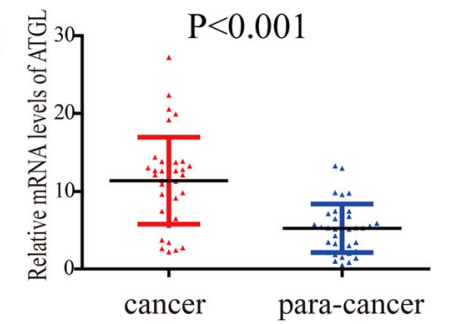

C

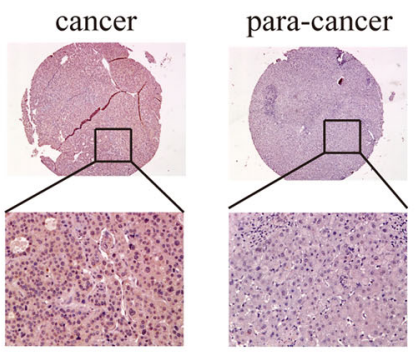

e
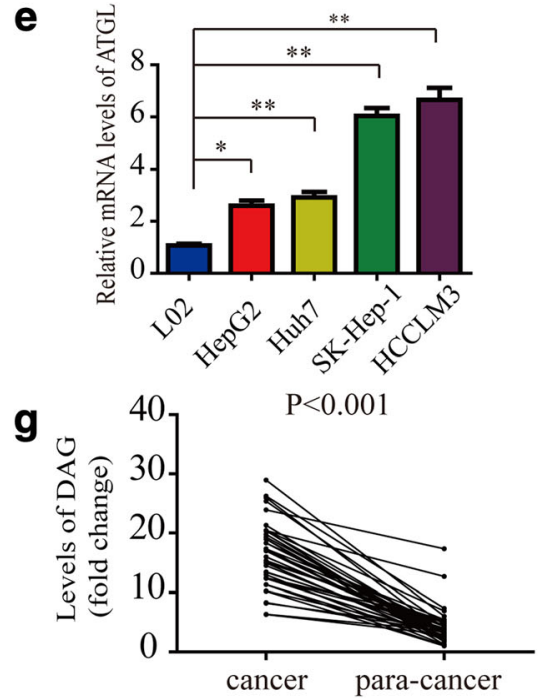

b

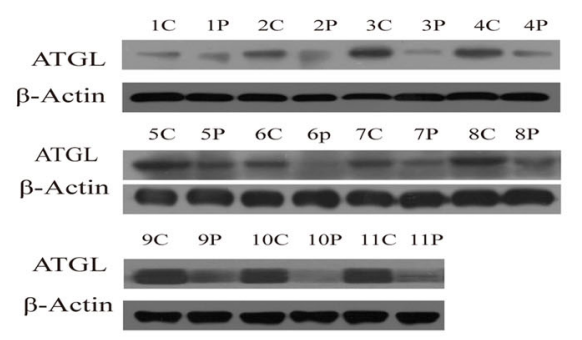

d

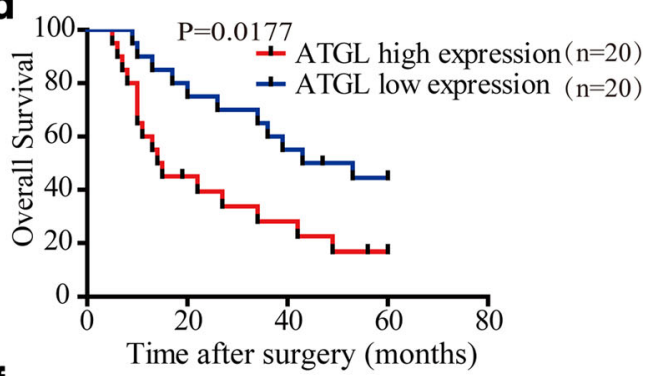

$\mathbf{f}$
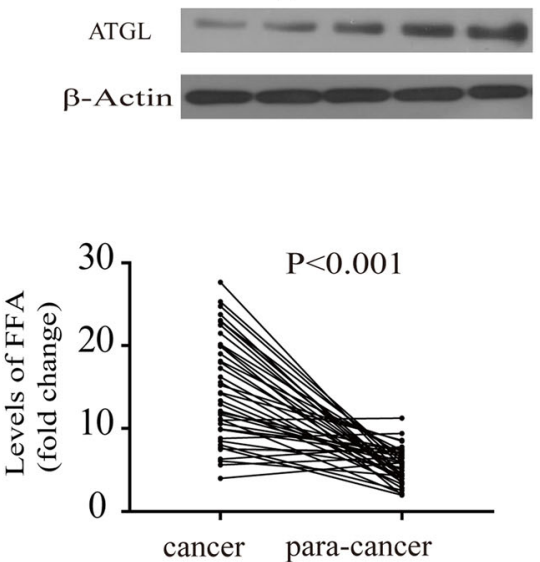

Fig. 1 ATGL is highly expressed in human HCC tissues and predicts poor prognosis. a Real-time PCR analysis of ATGL expression in 40 pairs of HCC and matched non-tumor tissues. b Western blot analysis of ATGL expression in HCC and matched non-tumor tissues C: cancer tissue; P: para-cancer tissue. c Representative immunohistochemical detection of ATGL in normal liver tissue and HCC tissues. $\mathbf{d}$ Kaplan-Meier analysis of overall survival in 40 patients indicated that high expression of ATGL predicts poor prognosis. The cutoff lines to divide into high and low group was median value. e Real-time PCR analysis of ATGL expression in HCC cell lines. $\mathbf{f}$ Western blot analysis of ATGL expression in HCC cell lines. $\mathbf{g}$ Relative levels of DAG and FFA in 40 pairs of HCC and matched non-tumorous tissues. Data are expressed as mean \pm SD of three independent experiments. Statistical significance was concluded at ${ }^{*} P<0.05,{ }^{*} P<0.01$

curves and CCK-8 assay results, sh-ATGL significantly inhibited HCC cell growth and this effect was completely rescued by treatment with $16 \mu \mathrm{M}$ DAG +FFA (Fig. 2c and Additional file 4: Figure S2D). Colony formation assay further confirmed this process (Fig. $2 \mathrm{~d}$ and Additional file 4: Figure S2E and F).

To determine whether these results were reproducible in vivo, we constructed an orthotopic xenografts HCC model in nude mice. Consistent with the results from in vitro assays, larger HCC tumors were observed in Huh7 overexpressing tumors compared with Huh7 control tumors (Fig. 2e-g). There were more
Ki-67 positive cells in Huh7 overexpressing tumors compared with Huh7 control tumors (Fig. 2h and Additional file 6: Figure S4A). To explore the role of DAG and FFA on tumor growth in vivo, we injected $20 \mathrm{mg} / \mathrm{kg}$ DAG+FFA intraperitoneally daily for 5 weeks. The impaired tumor growth rate of HCCLM3 sh-ATGL tumors was completely rescued in mice treated with DAG+FFA. Notably, treatment with DAG+FFA significantly promoted the growth of HCCLM3 sh-control tumors (Fig. 2i-k). Furthermore, the IHC results indicated that Ki-67 was significantly decreased in shATGL tumors and this effect was completely rescued 


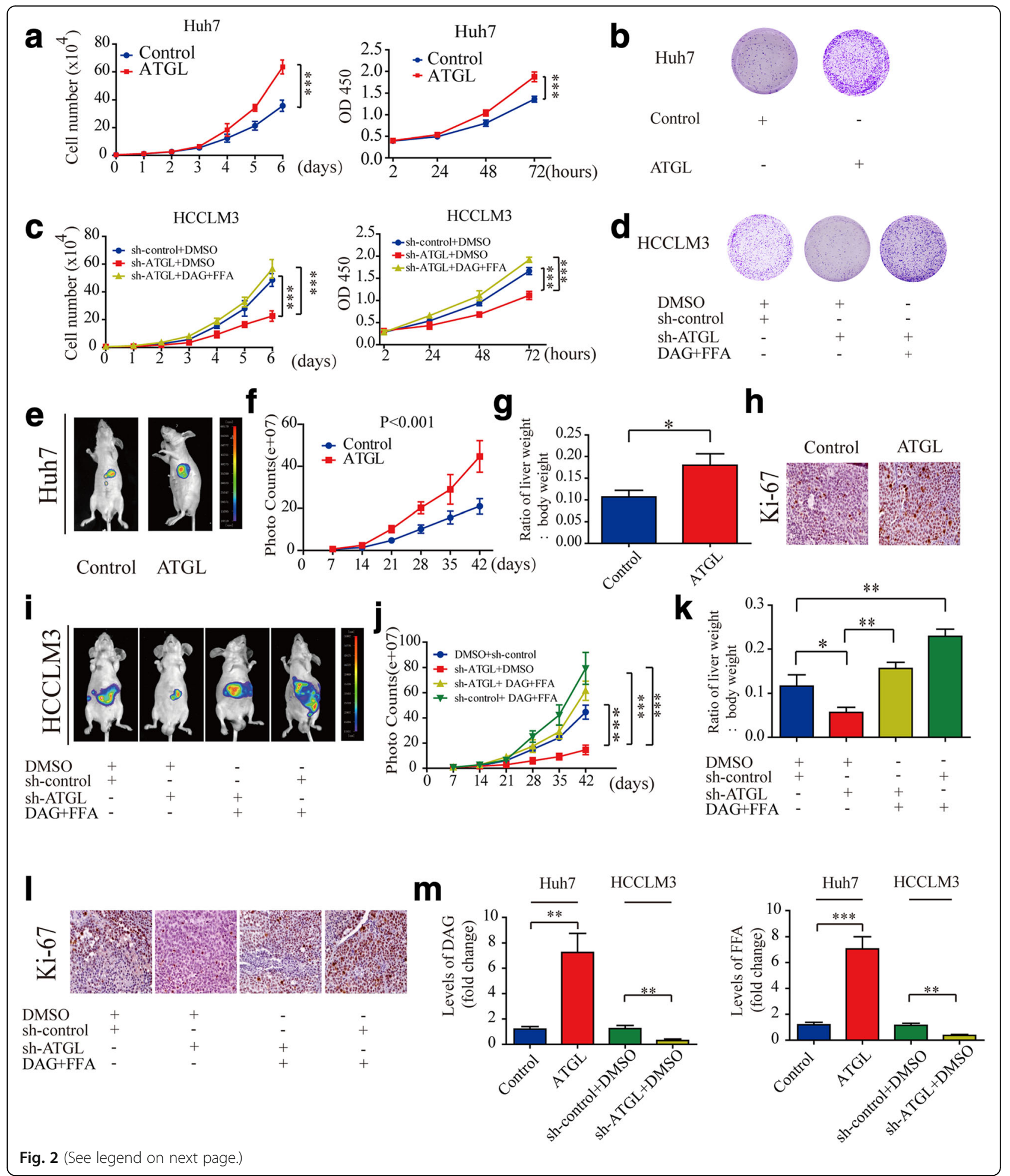




\begin{abstract}
(See figure on previous page.)
Fig. 2 ATGL promotes HCC cell growth by maintaining elevated levels of DAG and FFA in vitro and in vivo. a Growth curves for the indicated HCC cells were evaluated by the Trypan blue dye exclusion method (left panel). CCK-8 assays showed that overexpression of ATGL promoted the growth of Huh7 cells (right panels). $\mathbf{b}$ Representative images of the cloning formation assay showed that overexpression of ATGL promoted the growth of Huh7 cells. c Growth curves for the indicated HCC cells were evaluated by the Trypan blue dye exclusion method (left panel). CCK-8 assays showed that sh-ATGL significantly inhibited HCC cell growth, however, this effect was completely rescued by treatment with $16 \mu \mathrm{M}$ DAG +FFA (right panel). $\mathbf{d}$ Representative images of the cloning formation assay. showed that sh-ATGL significantly inhibited HCC cell growth and this effect was completely rescued by treatment with $16 \mu \mathrm{M}$ DAG +FFA. e Representative bioluminescence imaging of Huh7 orthotopic HCC tumors $\mathbf{f}$ Volume of Huh-7 orthotopic tumors was determined at different timepoints. $\mathbf{g}$ The ratio of liver weight/body weight. $\mathbf{h}$ Representative images of immunohistochemical detection of Ki-67 in orthotopic HCC tissues. i Representative bioluminescence imaging of HCCLM3 orthotopic HCC tumors. $\mathbf{j}$ Volume of HCCLM3 orthotopic tumors was determined at different timepoints. $\mathbf{k}$ The ratio of liver weight/body weight. I Representative images of immunohistochemical detection of Ki-67 in orthotopic HCC tissues. $\mathbf{m}$ Relative levels of DAG and FFA was determined in 100 mg orthotopic HCC tissues. Two-way ANOVA with the Bonferroni's correction was used in different timepoints statistical analysis. Data are expressed as mean \pm SD of three independent experiments. Statistical significance was concluded at ${ }^{*} P<0.05,{ }^{*} P<0.01,{ }^{* * *} P<0.001$
\end{abstract}

in mice treatment with DAG +FFA (Fig. $2 \mathrm{l}$ and Additional file 6: Figure S4B). In addition, levels of DAG and FFA in mice orthotopic $\mathrm{HCC}$ tissues were higher in the Huh7 overexpressing tumors than in the control tumors. In contrast, the silencing of ATGL expression had the opposite effects (Fig. 2m). In summary, these results indicated that ATGL promotes HCC cell growth by maintaining elevated levels of DAG and FFA in vitro and in vivo.

\section{LncRNA-NEAT1 modulates ATGL expression in HCC cells and disrupts the lipolysis of hepatoma cells via ATGL in vitro}

Recent publications outline a regulatory role for LncRNA in lipid metabolism [18]. Thus, we speculated that IncRNAs might modulate ATGL expression. To test this hypothesis, we screened for and identified six lncRNAs co-expressed with ATGL in liver cancer tissues using the online software tool Co-LncRNA (http://www. bio-bigdata.com/Co-LncRNA/) [19]. Then, we knocked down the expression of these lncRNAs using siRNA to examine their effect on ATGL in HCC cells. The transfection efficiencies were detected by qRT-PCR (Additional file 7: Figure S5A). Our results demonstrated $D A N C R$ knockdown up-regulated the expression of ATGL and that NEAT1 knockdown reduced the expression of ATGL in SK-Hep-1 cells (Fig. 3a). Given that resent studies has demonstrated that DANCR promotes HCC progression [20], and our study demonstrated that ATGL also promoted HCC cell growth. Therefore we pay attention to NEAT1. Next, we assessed NEAT1 levels in HCC cells with different growth potentials and found that NEAT1 RNA levels increased progressively from healthy liver cells to HCC cells with low growth potential and, finally, to HCC cells with high growth potential (Fig. 3b). Further studies revealed that knockdown of NEAT1 by lentiviral transfection down-regulated ATGL mRNA and protein levels in SK-Hep-1 and HCCLM3 cells (Fig. 3c). The transfection efficiency was detected by qRT-PCR (Additional file 7: Figure S5B). However, we did not observe alterations in MAGL and HSL at mRNA or protein levels (Additional file 8: Figure S6.A and B).

Next, we investigated the effect of NEAT1 on lipolysis in hepatoma cells. Reductions in intracellular FFA and DAG levels were observed following NEAT1 knockdown, however overexpression of ATGL blocks this phenomenon. Simultaneously, knockdown of NEAT1 with knockdown of ATGL (or treatment with Atglistatin) resulted in reduced DAG and FFA levels in HCCLM3 and SK-Hep-1 cells, however the effects were less than additive (Fig. $3 \mathrm{~d}$ and e).

To confirm NEAT1 was differentially expressed in HCC tissues, 40 pairs of HCC tissues and matching para-cancer tissues were used to evaluate NEAT1 expression. As expected the expression of NEAT1 was higher in HCC tissues than in the corresponding paracancer liver tissues (Fig. 3f). Furthermore, IHC staining results indicated that ATGL was significantly higher in HCC tissues with relatively high NEAT1 expression compared to that in tissues with relatively low NEAT1 expression (Fig. 3g). Moreover, we found that NEAT1 expression was positively correlated with tumor size. However, there was no correlation with HBV infection (Additional file 1: Table S2). In addition, NEAT1 expression was found to be positively correlated with ATGL, DAG and FFA levels in HCC tissues (Fig. 3h-j). In summary, our results revealed that the NEAT1 modulates ATGL expression in HCC cells and disrupts the lipolysis of hepatoma cells via ATGL.

\section{Abnormal lipolysis regulated by NEAT1-ATGL axis promotes $\mathrm{HCC}$ cell growth in vitro}

Next we evaluated the role of NEAT1 in HCC. According to growth curves and CCK- 8 assay results, we found that NEAT1 knockdown significantly inhibited the proliferation of HCC cells. This inhibitory effect was reversed by the overexpression of ATGL (Fig. 4a and b). Colony formation assay further confirmed this effect (Fig. 4c). Given that recent publications indicated that NEAT1 is transcriptionally regulated by the tumor suppressor p53(TP53) [21, 22], the survival effect of NEAT1 


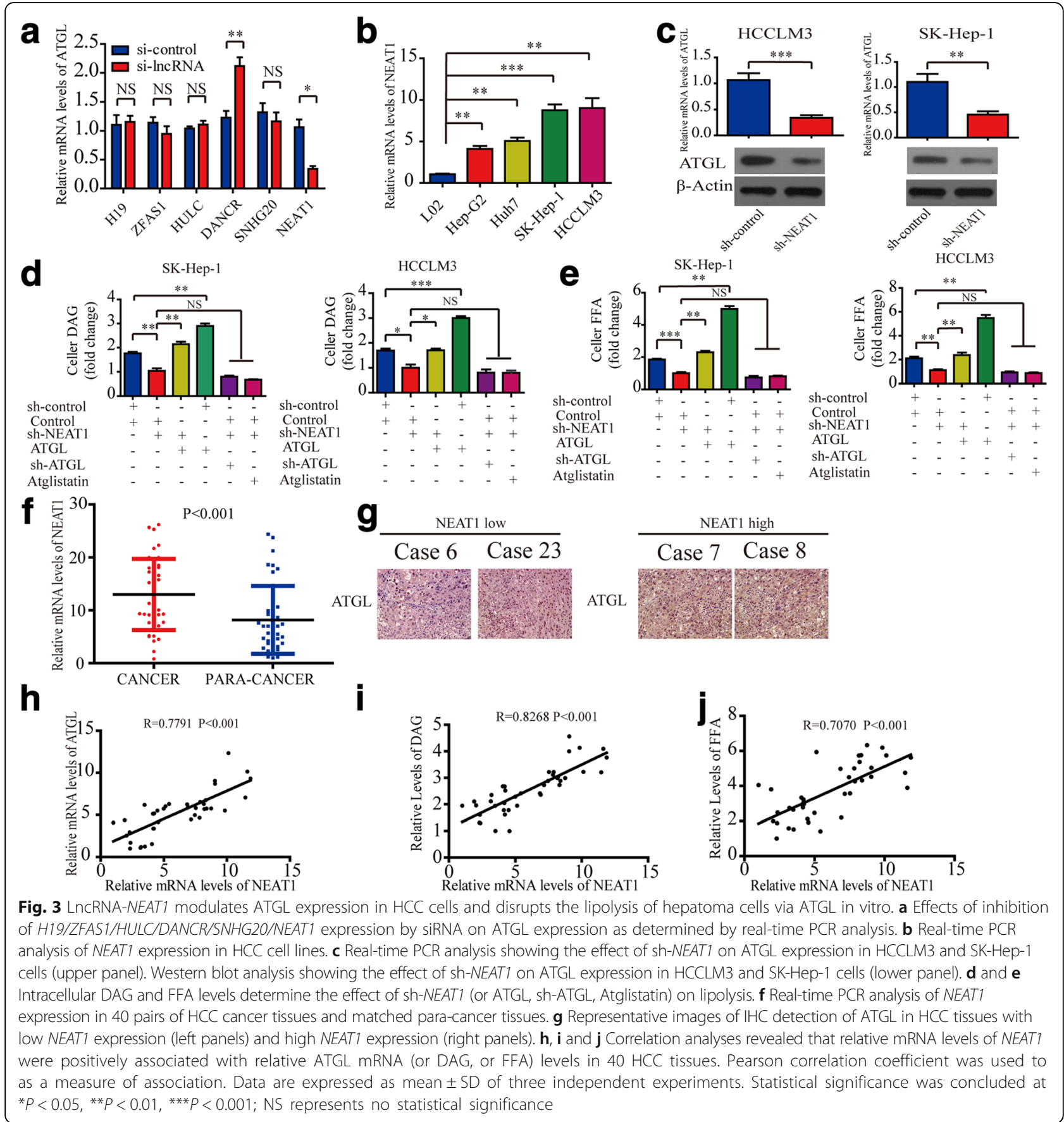

may alter across different cancers. We also tested the TP53 gene mutations in $40 \mathrm{HCC}$ tissues by PCR and direct sequencing. Given that exons 2-11 are coding exons of the p53 gene, we analyzed the sequence of p53 exons 2-11 for mutations. P53 mutations were found in 35\% HCC tissues(14/40). The p53 mutations were located in exons 4-9 (Additional file 2: Table S6). Consistent with the published reports, our results showed that the expression of NEAT1 was higher in TP53 wild-type tissues than in the TP-53 mutant liver tissues (Additional file 9: Figure S7A). Further, treatment with $10 \mu \mathrm{M}$ Nutlin-3a(TP53 stabilizer) for $24 \mathrm{~h}$ resulted in higher NEAT1 levels in TP53-wild-type Hep-G2 and SK-hep-1 cells but not in TP53 mutant Huh7 (p.Tyr220Cys) and HCCLM3 (p.Arg249Ser) cells (Additional file 9: Figure S7B). These results indicated that NEAT1 is a TP53 target gene in HCC. Given that a recent publication reported that NEAT1 expression can in turn prevent accumulation of TP53 [21]. We tested TP53 expression in NEAT1 knockdown cells. 


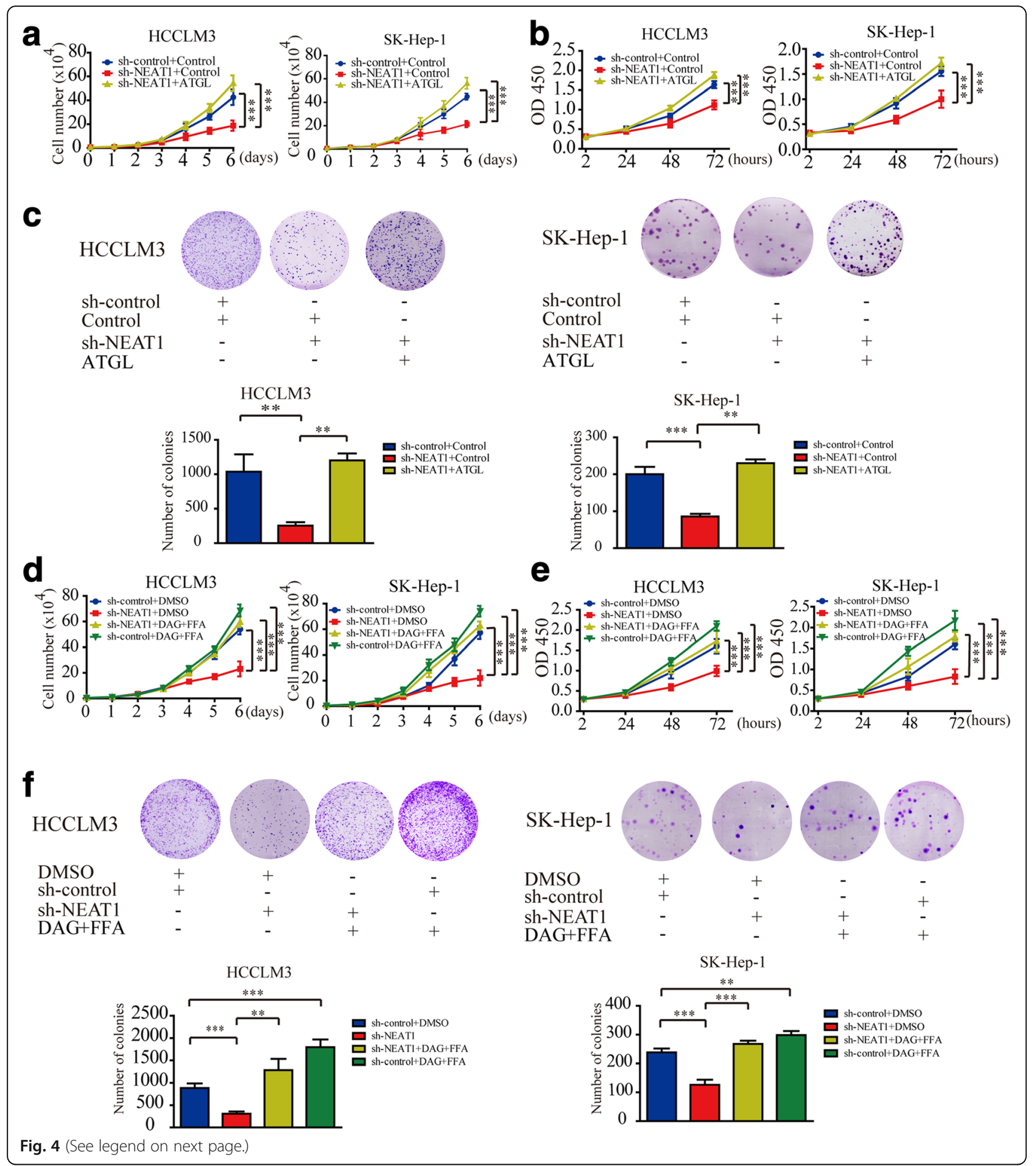




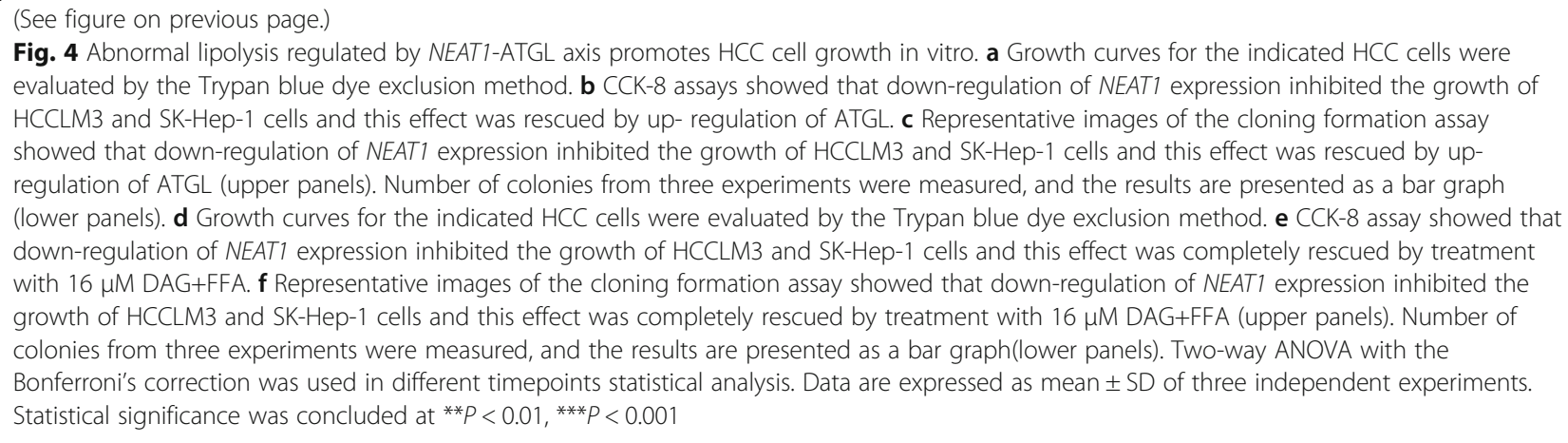
evaluated by the Trypan blue dye exclusion method. b CCK-8 assays showed that down-regulation of NEAT1 expression inhibited the growth of HCCLM3 and SK-Hep-1 cells and this effect was rescued by up- regulation of ATGL. c Representative images of the cloning formation assay showed that down-regulation of NEAT1 expression inhibited the growth of HCCLM3 and SK-Hep-1 cells and this effect was rescued by upregulation of ATGL (upper panels). Number of colonies from three experiments were measured, and the results are presented as a bar graph (lower panels). $\mathbf{d}$ Growth curves for the indicated HCC cells were evaluated by the Trypan blue dye exclusion method. e CCK-8 assay showed that down-regulation of NEAT1 expression inhibited the growth of HCCLM3 and SK-Hep-1 cells and this effect was completely rescued by treatment with $16 \mu \mathrm{M}$ DAG+FFA. $\mathbf{f}$ Representative images of the cloning formation assay showed that down-regulation of NEAT1 expression inhibited the growth of HCCLM3 and SK-Hep-1 cells and this effect was completely rescued by treatment with $16 \mu \mathrm{M}$ DAG+FFA (upper panels). Number of colonies from three experiments were measured, and the results are presented as a bar graph(lower panels). Two-way ANOVA with the Bonferroni's correction was used in different timepoints statistical analysis. Data are expressed as mean \pm SD of three independent experiments. Statistical significance was concluded at ${ }^{* *} P<0.01,{ }^{* * *} P<0.001$

Consistent with the previous report, TP53 was upregulated following NEAT1 knockdown (Additional file 9: Figure S7C). To confirm this phenomenon, we also evaluated the expression of $\mathrm{p} 21$ and Bax, the target gene of $\mathrm{p} 53$. The results indicated that $\mathrm{p} 21$ and Bax was upregulated following NEAT1 knockdown in HCC cell (Additional file 9: Figure S7D). These results indicated that NEAT1 is a TP53 target gene in HCC and plays an oncogenic role in HCC. Further, ATGL is responsible for NEAT1-mediated HCC cell growth in vitro.

Considering that the pro-tumorigenic effects of ATGL were mediated by DAG and FFA, we hypothesized that the effect of NEAT1 on HCC cell growth was also mediated by DAG and FFA. We found that NEAT1 knockdown significantly inhibited the proliferation of HCC cells. This inhibitory effect was reversed by treatment with $16 \mu \mathrm{M}$ DAG+FFA. Notably, treatment with DAG+FFA significantly promoted HCC cell growth (Fig. 4d-f).

Thus, we concluded ATGL and its products, DAG and FFA, are responsible for NEAT1-mediated HCC cell growth. Abnormal lipolysis regulated by NEAT1-ATGL axis promotes $\mathrm{HCC}$ cell growth in vitro.

\section{NEAT1-regulated abnormal lipolysis facilitates HCC cell growth in vivo}

To better understand the hepatocarcinogenesis role of NEAT1-regulated abnormal lipolysis, we established orthotopic xenografts HCC model in nude mice. The results revealed that the inhibition of NEAT1 expression led to smaller HCC tumors than control tumors, but this effect was blocked by co-transfection of sh$N E A T 1+$ ATGL. Additionally, sh-NEAT1 + ATGL tumors were smaller compared with ATGL overexpression tumors (Fig. 5a-c). Further, the IHC results indicated that Ki-67 and ATGL was significantly decreased in sh-NEAT1 group and this effect was rescued in mice co-transfection of sh-NEAT1 + ATGL (Fig. 5d). We then determined whether increased DAG+FFA delivery could rectify the tumor growth defect observed in sh-NEAT1 cells in vitro. Orthotopic xenografts mice were injected with $20 \mathrm{mg} / \mathrm{kg}$ DAG
+FFA intraperitoneally daily for 5 weeks. The impaired tumor growth rate of sh-NEAT1 tumors was completely rescued in mice injected with DAG+FFA. Notably, treatment with DAG+FFA significantly promoted HCCLM3 sh-control tumor growth (Fig. 5e-g). Ki-67 IHC staining further confirmed this effect (Fig. 5h). In addition, levels of DAG and FFA in mice with orthotopic HCC tissues were lower in the sh-NEAT1 tumors than in the control tumors, however, this effect was reversed by co-transfection of sh-NEAT1 + ATGL (Fig. $5 \mathrm{i}$ and $\mathrm{j}$ ). Taken together, these results indicate that NEAT1-regulated abnormal lipolysis contributes to HCC growth, and that this process is mediated by ATGL and its product, DAG+FFA, in vivo.

\section{Knockdown of NEAT1 down-regulates ATGL expression by upregulating miR-124-3p levels}

Several studies have confirmed that microRNAs are important target of IncRNAs [23, 24]. To identify whether any microRNAs represent potential targets that can be bind to ATGL and NEAT1, we used three online software tools (targetscan: http://www.targetscan.org/; microrna. org: http://www.microrna.org/; Starbase: http://starbase. sysu.edu.cn/.) to identify potential binding sites. We identified four microRNAs (hsa-miR-103a-3p, hsa-miR214-3p, hsa-miR-124-3p and hsa-miR-107) that exhibited potential to bind to both NEAT1 and ATGL. MiR-124-3p, which had the highest alignment score/Pct score is reported to have anti-cancer effects, therefore, we focused our investigation on this miRNA. The predictive information between miR-124-3p and the binding sites in the NEAT1/ATGL 3'-UTRs was illustrated in Fig. 6a. We further analysed the minimum free energy value of the hybrid between miR-124-3p and the binding site on the NEAT1/ATGL 3'-UTRs. The minimum free energy values were $-14.6 \mathrm{kcal} / \mathrm{mol}$ and $-17.5 \mathrm{kcal} / \mathrm{mol}$, respectively, which are within the range of genuine miRNA-target pairs. Given the fact that miRNAs can target the nuclear IncRNA [25, 26], we first explored the subcellular distribution of miR-124-3p by FISH. The results showed that miR-124-3p was present both in the nucleus and 


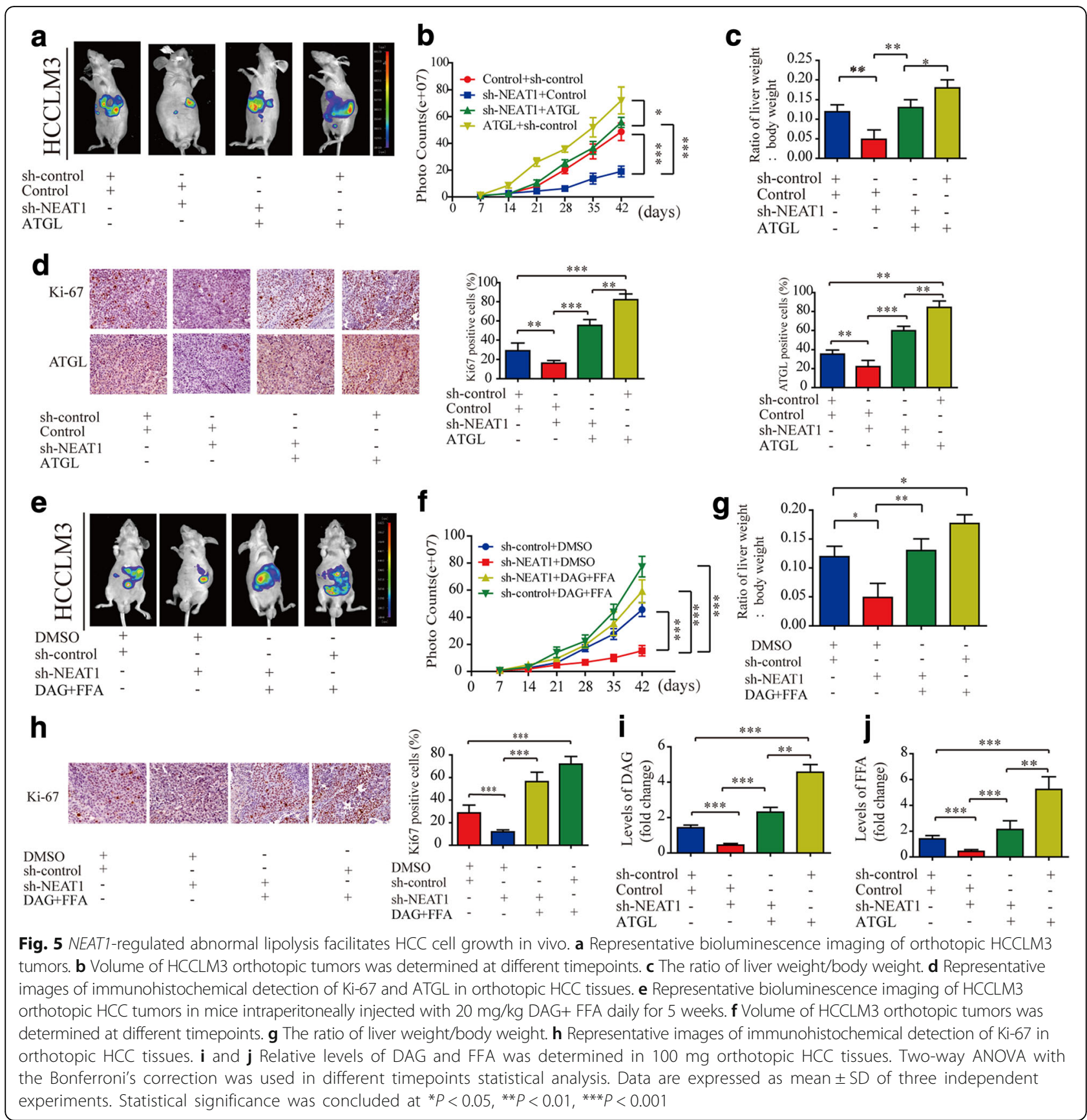

cytoplasm (Additional file 10: Figure.S8A). Next we explored that whether NEAT1 is able to regulate miR-124$3 \mathrm{p}$ expression. The results indicated that NEAT1 knockdown led to a significant increase in miR-124-3p expression in HCC cells as assayed by qRT-PCR (Fig. 6b). In addition, a dual-luciferase reporter assay demonstrated that miR-124-3p mimics decreased the luciferase activities of NEAT1-WT but failed to influence the mutant, suggesting that miR-124-3p is able to directly bind to the NEAT1-WT target sites in $293 \mathrm{~T}$ cells (Fig. 6c). These results prove that NEAT1 regulate miR-124-3p expression in a sequence-specific and binding-dependent manner. Next, we overexpressed miR-124-3p using a miR-124$3 p$ mimic. Western blot and qRT-PCR results indicated that exogenous miR-124-3p significantly reduced ATGL expression in HCCLM3 and SK-Hep-1 cells (Fig. 6d). Our previous results demonstrated that knockdown of NEAT1 down-regulated ATGL expression, and we found that this suppression can be attenuated in HCC cells by inhibiting miR-124-3p (Fig. 6e). The transfection efficiencies of miR-124-3p were detected by qRTPCR (Additional file 11: Figure S9A). In addition, a dual- 


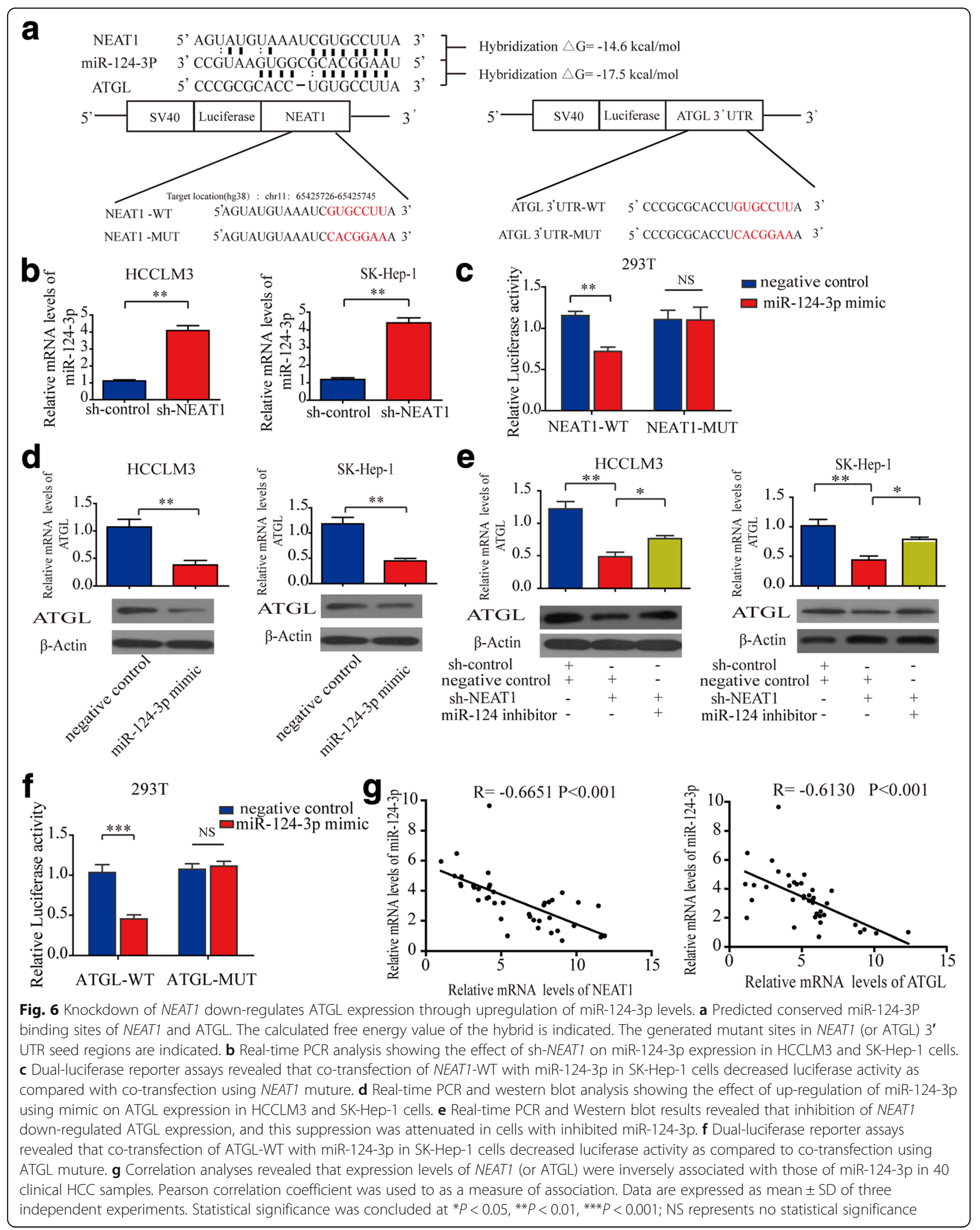


luciferase reporter assay demonstrated that exogenous miR-124-3p reduced the luciferase activity of ATGL-WT but did not affect the luciferase activity of ATGL-MUT in $293 \mathrm{~T}$ cells (Fig. 6f). To further prove that miR-124-3p is involved in the cross-regulation between NEAT1 and ATGL, We performed a rescue experiment with a dualluciferase reporter assay using ATGL with mutant miR124-3p binding sites. The results showed that depletion of NEAT1 in $293 \mathrm{~T}$ cells inhibited the luciferase activity of ATGL-WT but not ATGL-MUT. Further, inhibition of miR-124-3p reversed this decrease in luciferase activity for ATGL-WT, but not for ATGL-MUT (Additional file 12: Figure S10A). Correlation analyses revealed that expression levels of NEAT1 and ATGL were inversely associated with that of miR-124-3p in 40 clinical HCC samples (Fig. $6 \mathrm{~g}$ ). These results support the idea that NEAT1 regulates ATGL expression through miR-124-3p.

To confirm the effect of miR-124-3p on lipolysis, we overexpressed miR-124-3p using miR-124-3p mimics in HCC cell lines. Our results indicated that both DAG and FFA levels were decreased in HCC cells (Additional file 13: Figure S11A and B). We also evaluated NEAT1 and miR-124-3p expression in five pairs of HCC and matched non-tumor tissues by qRT-PCR. NEAT1 was upregulated and miR-124-3p was downregulated in the five $\mathrm{HCC}$ tissues compared with the matched nontumor tissues (Additional file 14: Figure S12A).

\section{Knockdown of NEAT1 attenuates HCC cell growth through miR-124-3p/ ATGL/ DAG+FFA/ PPARa signaling}

Considering that cancer cells have been reported to increase fatty acid oxidation (FAO) for cell survival due to compromised glucose uptake and ATGL-PPAR $\alpha$ signaling have been reported to mediate FAO [27, 28], we hypothesized that NEAT1 might serve as a means to mediate FAO through PPAR $\alpha$. To test this hypothesis, we first tested whether ATGL mediated PPAR $\alpha$ in HCC cells. Our results showed that overexpression of ATGL significantly up-regulated PPAR $\alpha$ expression in HCC cells (Fig. $7 \mathrm{a}$ and b). As various studies using a variety of biochemical techniques have firmly corroborated the direct physical association between fatty acids and PPAR $\alpha$ and have thus established fatty acids as bona fide PPAR $\alpha$ ligands [29], we hypothesized that DAG or FFA may active PPAR $\alpha$ expression. To examine whether DAG+FFA regulates PPAR $\alpha$ expression, we treated HCC cells with $16 \mu \mathrm{M}$ and $32 \mu \mathrm{M}$ DAG+FFA for $36 \mathrm{~h}$. The resulting data demonstrated that DAG + FFA up-regulates the expression of PPAR $\alpha$ in Huh7 and HCCLM3 cells in a dose dependent manner (Fig. 7c). In addition, our results showed that knockdown of NEAT1 reduced PPAR $\alpha$ levels, however, treatment with miR-124-3p inhibitor (or overexpression of ATGL/treatment with $16 \mu \mathrm{M}$ DAG+FFA) blocked this process (Fig. 7d). The transfection efficiencies of miR-124-3p were detected by qRT-PCR (Additional file 11: Figure S9B). PPAR $\alpha$ is a known oncogene in HCC [30]. Thus, we concluded that NEAT1 mediates HCC cell growth via miR-124-3p/ATGL/DAG+FFA/PPAR $\alpha$ signaling. Moreover, NEAT1 may mediate FAO via PPAR $\alpha$, though this effect requires further study in the future.

\section{Combination of NEAT1 and ATGL exhibits improved prognostic accuracy for HCC}

Our results indicated NEAT1 expression was negatively correlated with patient survival (Fig. 8a). It was previously shown that a combination of molecular markers can improve the prediction of patient prognosis [31]. We investigated whether this was the case for NEAT1 and ATGL in HCC patients. Indeed, patients with tumors exhibiting high levels of NEAT1 and high levels of ATGL had lower survival (Fig. 8b). Thus, combining NEAT1 and ATGL expression improves the prediction of patient outcome.

\section{Discussion}

In this study, we demonstrated that the NEAT1/miR-1243p/ATGL pathway plays an important role in regulating abnormal lipolysis in HCC. In addition, NEAT1-mediated abnormal lipolysis facilitates HCC cell growth in vivo and in vitro.

Recent studies have demonstrated that fatty acids(FAs) may contribute to cancer progression through multiple mechanisms. Given that cancer cells can acquire FAs for their growth and proliferation through lipogenesis and lipolysis [4], we speculated that lipolysis may play a vital role in HCC development. Our results indicated that the lipolytic enzyme ATGL is highly expressed in HCC tissues and predicts poor prognosis. Although present studies have revealed that inhibition of ATGL attenuated the growth and motility of tumor cells [8], our study answered an important question: whether ATGL mediates lipolytic metabolic are responsible for this process. Our experimental results indicate that the pro-tumorigenic effects of ATGL were mediated by its DAG and FFA products. In addition, our results serve to explain, at least in part, high levels of DAG and FFA present in HCC tissues.

Recent publications outline a regulatory role for LncRNA in lipid metabolism [32], however, whether IncRNAs can influence the development of cancer through participating in lilpolytic metabolic, and the biochemical pathways involved in this process are both unknown. For this, we identified lncRNAs that are co-expressed with ATGL through the online tool Co-lncRNA. Based on this analysis, our results demonstrated that the down-regulation of NEAT1 expression attenuates ATGL expression in HCC cells. In addition, we showed that NEAT1 expression was positively correlated with ATGL levels in HCC tissues. These results indicated that NEAT1 modulates ATGL expression in HCC. Further, reductions in intracellular FFA and DAG 


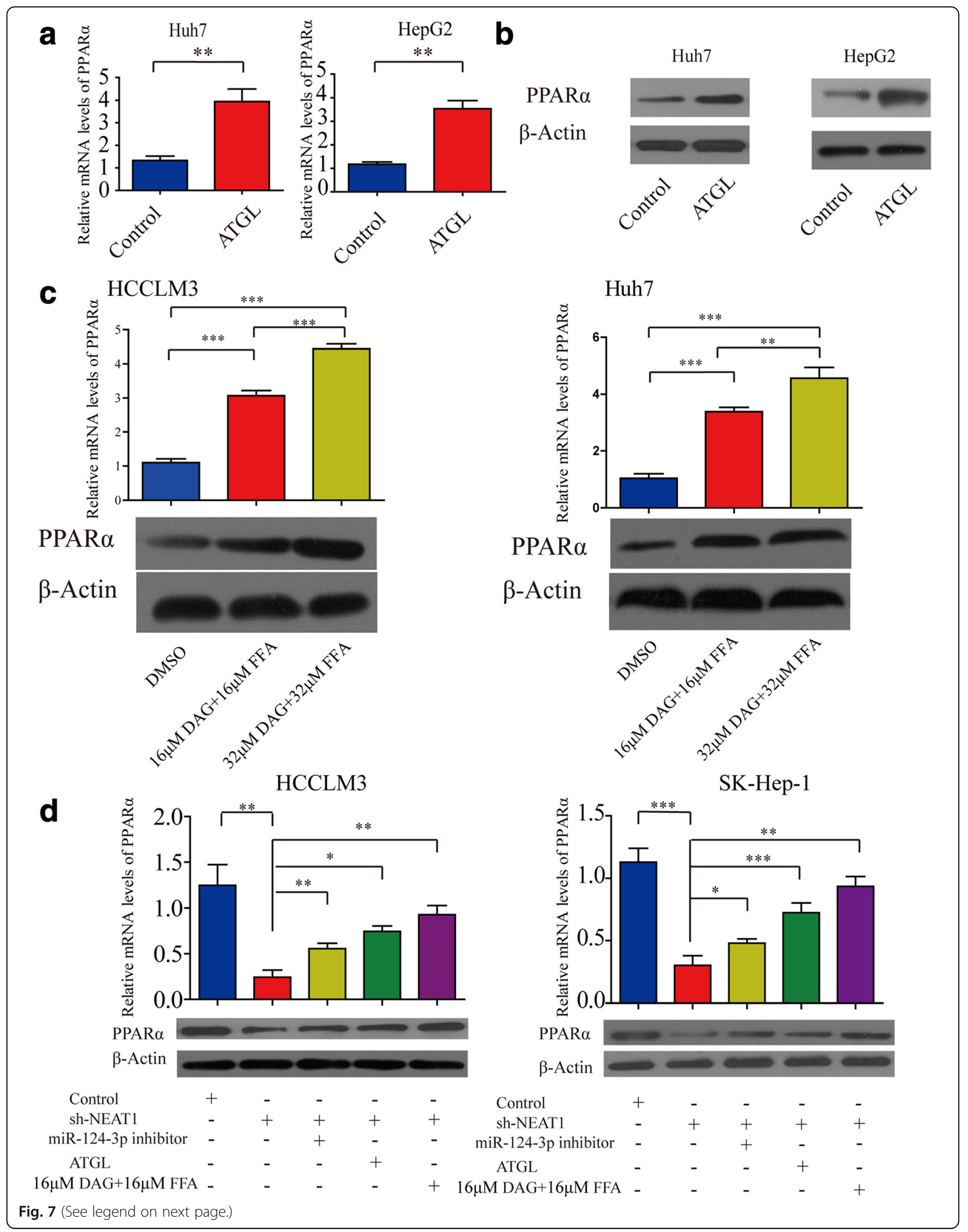


(See figure on previous page.)

Fig. 7 Knockdown of NEAT1 attenuates HCC cell growth through miR-124-3P/ ATGL/ DAG+FFA/ PPARa signaling. a Real-time PCR analysis showing the effect of up-regulation of ATGL on PPARa expression in Huh7 and HepG2 cells. $\mathbf{b}$ Western blot analysis showing the effect of up-regulation of ATGL on PPARa expression in Huh7 and HepG2 cells. c Real-time PCR and western blot showing the effect of treatment with $16 \mu \mathrm{M}$ and $32 \mu \mathrm{M}$ DAG+FFA on PPARa expression in Huh7 and HCCLM3 cells. $\mathbf{d}$ Real-time PCR analysis and western blot analysis revealed that the sh-NEAT1 down-regulated PPARa expression in HCCLM3 and SK-Hep-1 cells, whereas miR-124-3p inhibitor treatment (or overexpression of ATGL/treatment with DAG+FFA) blocked this process. Data are expressed as mean \pm SD of three independent experiments. Statistical significance was concluded at ${ }^{*} P<0.05,{ }^{* *} P<0.01,{ }^{* * *} P<0.001$

levels were observed following NEAT1 knockdown. Our results demonstrated that NEAT1 disrupts the lipolysis of hepatoma cells via ATGL. Notably, we determined that ATGL and its products, DAG and FFA, are responsible for NEAT1 mediated HCC cell growth. These results demonstrated that NEAT1-modulated abnormal lipolysis promotes HCC cell growth in vivo and in vitro, providing new insight into the mechanism of HCC development.

Recent studies have been demonstrated that NEAT1 is important in Adipogenesis [33]. Our results indicated that NEAT1 also mediates lipolysis in HCC cells, indicating that NEAT1 may be a central regulator in lipid metabolism. Other genes play similar central roles in lipid metabolism, including those encoding mTOR, PPARs (peroxisome proliferator-activated receptors), TNF-alpha and SIRT1 [34-37]. Further studies are needed to identify the effect of crosstalk between NEAT1 and these genes.

NEAT1 is up-regulated in various types of cancers and several studies have indicated multiple mechanisms of NEAT1 up-regulation. First, the activation of hypoxia pathways is a feature of HCC. Previous studies indicated that NEAT1 expression is upregulated by hypoxia through HIF-2 $\alpha$ [38]. Second, NEAT1 is also regulated by microRNAs. The findings suggest that the microRNA-NEAT1 regulatory network plays significant cellular and physiological roles, and that its dysregulation contributes to tumorigenesis [39]. Third, Some transcription factors such as Oct4 and estrogen receptor alpha have been reported
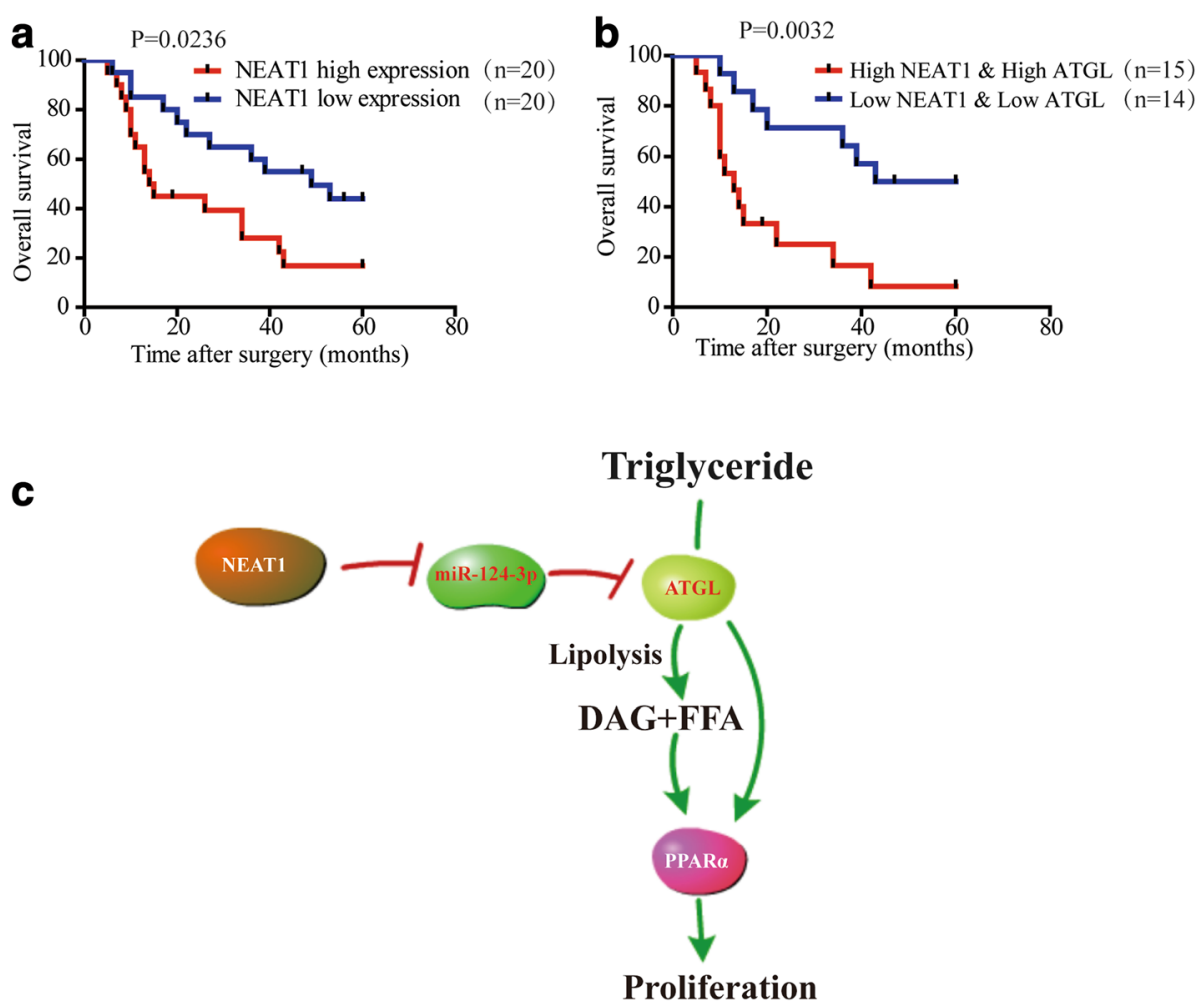

Fig. 8 Combination of NEAT1 and ATGL exhibits improved prognostic accuracy for HCC. a Kaplan-Meier analysis of overall survival in 40 patients indicated that high expression of NEAT1 predicts poor prognosis. The cutoff lines to divide into high and low group was median value. $\mathbf{b}$ Kaplan-Meier analysis of overall survival in 40 patients indicated that patients with tumors exhibiting high levels of NEAT1 and high levels of ATGL had lower survival. The cutoff lines to divide into high and low group was median value. c Schematic presentation of the mechanism underlying NEAT1-regulated abnormal lipolysis in hepatocarcinogenesis. NEAT1 regulates ATGL expression via directly bind to miR-124-3p. NEAT1 can influence the HCC proliferation through participating in lipolysis via ATGL. ATGL and its products, DAG and FFA, are responsible for NEAT1-mediated HCC cell growth through PPARa activation 
to regulate $N E A T 1$ expression by directly binding NEAT1 promoters [40, 41]. However, NEAT1 is down-regulated and plays a tumor-suppressor role in specific cancer types, because NEAT1 is a target gene of wild type p53 [22, 4244].. These seemingly contradictory results may reflect cell type-specific roles for NEAT1 in tumorigenesis. An important mechanism that can explain this discrepancy is that NEAT1 can in turn prevent accumulation of TP53 [21]. Our result confirmed this phenomenon in HCC cell lines. NEAT1 inhibits wild type p53 tumor suppressive functions through this negative feedback loop. However, whether this negative feedback loop is a universal phenomenon in human cancers needs further study. Additionally, P53 is the most commonly mutated gene in human cancers. According to the literature, NEAT1 is also highly expressed in the mutant p53 cell lines, such as lung cancer cell line: H1299; breast cancer cell line: MDA-MB231; pancreatic cancer cell line PACN-1, colon cancer cell line: SW480 [12, 45-47]. This indicated some other NEAT1 regulator may play an important role in NEAT1 expression. Studies have revealed that some tumorpromoters, such as HIF-2, Oct4, and estrogen receptor alpha, can strongly enhance NEAT1 expression [38, 40, 41]. NEAT1 could participate in the related gene pathway mentioned above to strongly promote tumor development.

Several studies have indicated that microRNAs can be important targets of lncRNAs [23, 24]. In this study, our results indicated that the interaction of NEAT1 with ATGL might occupy the binding site of miRNAs so that suppression of ATGL by miR-124-3p would be significantly retarded. Meanwhile, dual-luciferase reporter assays demonstrated that both NEAT1 and ATGL directly bind to miR-124-3p. We speculate that there may be a novel regulatory transcript-mediated release of ATGL from miRNA repression which would add to the known crosstalk within the established pathway.

To elucidate the mechanism of NEAT1-modulated abnormal lipolysis in hepatocarcinogenesis, we examined the expression of PPAR $\alpha$, the downstream target of ATGL [48]. PPAR $\alpha$ is expressed mainly in the liver, heart, and muscles. It is a major regulator of fatty acid transport, catabolism, and energy homeostasis [49]. PPAR $\alpha$ plays an important role in HCC proliferation [26]. Our results showed that ATGL expression enhances PPAR $\alpha$ levels in HCC cells. Interestingly, our results also showed that treatment with DAG + FFA up-regulates the expression of PPAR $\alpha$ in Huh7 and HCCLM3 cells in a dose dependent manner. Importantly, the knockdown of NEAT1 down-regulated PPAR $\alpha$ expression, but this process could be blocked by treatment with miR-124-3p inhibitor (or overexpression of ATGL/treatment with DAG+FFA). Therefore, we conclude that NEAT1 promotes HCC cell growth through miR-124-3p/ ATGL/DAG+FFA/PPAR $\alpha$ signaling.

\section{Conclusions}

In summary, we find that the lncRNA-NEAT1 disrupts HCC cell lipolysis through ATGL. NEAT1 regulates ATGL expression via competitively binding to miR-1243p. Our results explain the high levels of DAG and FFA present in HCC tissues. ATGL and its products, DAG and FFA, are responsible for NEAT1-mediated HCC cell growth. Additionally, NEAT1 mediates HCC cell growth through the miR-124-3p/ATGL/DAG+FFA/PPAR $\alpha$ pathway. Importantly, Combination of NEAT1 and ATGL exhibits improved prognostic accuracy for HCC. Thus, we here demonstrate that NEAT1-modulated abnormal lipolysis promotes $\mathrm{HCC}$ cell growth.

\section{Additional files}

Additional file 1: Table S1. Relationship between ATGL expression and clinicopathological features of HCC patients. Table S2. Relationship between NEAT1 expression and clinicopathological features of HCC patients. (DOCX $20 \mathrm{~kb}$ )

Additional file 2: Table S3. Sequences of siRNAs used in this study. Table S4. Sequences of primers used in this study. Table S5. Primers design of the tp53 gene. Table S6. Tp53 mutational analysis by PCR and direct sequencing. (DOCX $20 \mathrm{~kb}$ )

Additional file 3: Figure S1. ATGL mRNA was aberrantly expressed in HCC tissues and mediates lipolysis in HCC cells. A. Real-time PCR analysis of HSL, MAGL and ATGL expression in five pairs of HCC and matched non-tumor tissues. B. Transfection efficiency of ATGL and sh-ATGL as detected by western blot. C. Overexpression of ATGL increased intracellular FFA and DAG levels in Huh7 and HepG2 cell lines. D. ATGL knockdown (or treatment with Atglistatin) reduced intracellular FFA and DAG levels in HCCLM3 and SK-Hep-1 cell lines. Data are expressed as mean \pm SD of three independent experiments. Statistical significance was concluded at ${ }^{* *} P<0.01,{ }^{* *} P<0.001$. (TIF $436 \mathrm{~kb}$ )

Additional file 4: Figure S2. ATGL promotes HCC cell growth in vitro. A. Growth curves for the indicated HCC cells were evaluated by the Trypan blue dye exclusion method (left panel). CCK-8 assays showed that overexpression of ATGL promoted the growth of HepG2 cells (right panel). B. Representative images of the cloning formation assay showed that overexpression of ATGL promoted the growth of HepG2 cells. C. Number of colonies from three experiments were measured, and the results are presented as a bar graph. D. Growth curves for the indicated HCC cells were evaluated by the Trypan blue dye exclusion method (left panel). CCK-8 assays showed that ATGL knockdown inhibited the growth of SK-Hep-1 cells (right panel), however, this effect was completely rescued by treatment with $16 \mu \mathrm{M}$ DAG+FFA. E. Representative images of the cloning formation assay showed that ATGL knockdown inhibited the growth of SK-Hep-1 cells, however, this effect was completely rescued by treatment with $16 \mu \mathrm{M}$ DAG+FFA. F. Number of colonies from three experiments were measured, and the results are presented as a bar graph. Data are expressed as mean \pm SD of three independent experiments. Statistical significance was concluded at ${ }^{*} P<0.05,{ }^{*} P<0.01$, ${ }^{* *} P<0.001$. (TIF $614 \mathrm{~kb}$ )

Additional file 5: Figure S3. Treatment with DAG and FFA promote HCC cell growth. A. CCK-8 assays determined the effect of treatment with DAG at the concentrations of $8 \mu \mathrm{M}, 16 \mu \mathrm{M}$, and $32 \mu \mathrm{M}$ on HCC cell growth. B. CCK-8 assays determined the effect of treatment with FFA at the concentrations of $8 \mu \mathrm{M}, 16 \mu \mathrm{M}$, and $32 \mu \mathrm{M}$ on HCC cell growth. C. CCK8 assays determined the effect of treatment with DAG, FFA, or DAG+FFA at a concentration of $16 \mu \mathrm{M}$ on HCC cell growth. Data are expressed as mean \pm SD of three independent experiments. Statistical significance was concluded at ${ }^{*} P<0.05,{ }^{* *} P<0.01,{ }^{* *} P<0.001$. (TIF $986 \mathrm{~kb}$ )

Additional file 6: Figure S4. Ki-67 positive cells in IHC. A. More Ki-67 positive cells in Huh7 overexpressing tumors compared with Huh7 control tumors B. Ki-67 positive cells were decreased in sh-NEAT1 tumors, 
however this effect was completely rescued in mice tumors injected DAG + FFA. Data are expressed as mean \pm SD. Statistical significance was concluded at ${ }^{*} P<0.01,{ }^{* * *} P<0.001$. (TIF $682 \mathrm{~kb}$ )

Additional file 7: Figure S5. Transfection efficiency as detected by qRTPCR. A. Transfection efficiency of H19/ZFAS1/HULC/DANCR/SNHG20/NEAT1 in SK-Hep-1 cells as detected by qRT-PCR. B. Transfection efficiency of NEAT1 as detected by qRT-PCR. C. Transfection efficiency of sh-NEAT1 and sh-ATGL in Fig. 3d, e as detected by western blot and qRT-PCR. Data are expressed as mean \pm SD of three independent experiments. Statistical significance was concluded at ${ }^{* * *} P<0.001$. (TIF $343 \mathrm{~kb}$ )

Additional file 8: Figure S6. NEAT1 does not mediate MAGL or HSL expression in HCC cells. A. Real-time PCR analysis determined the effects of sh-NEAT1 on MAGL and HSL in HCC cells. B. Western blot analysis determined the effect of sh-NEAT1 on MAGL and HSL in HCC cells. Data are expressed as mean \pm SD of three independent experiments. NS represents no statistical significance. (TIF $588 \mathrm{~kb}$ )

Additional file 9: Figure S7. NEAT1 is a TP53 target gene in HCC. A. The expression of NEAT1 was higher in TP53 wild-type tissues $(n=26)$ than in the TP-53 mutant liver tissues $(n=14)$. B. Treatment with Nutlin-3a resulted in higher NEAT1 levels in TP53 wild-type Hep-G2 and SK-hep-1 cells but not in TP53 mutant Huh7 and HCCLM3 cells. C. Western blot analysis determined TP53 was upregulated following NEAT1 knockdown in SK-Hep-1 and Hep-G2 cells. D. Western blot analysis determined p21 and Bax was upregulated following NEAT1 knockdown in SK-Hep-1 and Hep-G2 cells. Data are expressed as mean \pm SD of three independent experiments. Statistical significance was concluded at ${ }^{*} P<0.05,{ }^{*} P<0.01$, NS represents no statistical significance. (TIF $530 \mathrm{~kb}$ )

Additional file 10: Figure S8. The subcelluar distribution of miR-124-3p was explored by FISH. A. Representative images showing localization of CY3-miR-124-3p in HCC cell lines. 18S, probe for 18S rRNA; U6, probe for U6 snRNA. (TIF $739 \mathrm{~kb})$

Additional file 11: Figure S9. The transfection efficiencies of miR-124-3p were detected by qRT-PCR. A. The mRNA levels of miR-124-3p in Fig. 6e as detected by qRT-PCR. B The mRNA levels of miR-124-3p in Fig. $7 d$ as detected by qRT-PCR. Data are expressed as mean \pm SD of three independent experiments. Statistical significance was concluded at ${ }^{* * *}<0.001$. (TIF $820 \mathrm{~kb}$ )

Additional file 12: Figure S10. Dual-luciferase reporter assays reveals miR-124-3p is involved in the crossregulation between NEAT1 and ATGL. A. Dual-luciferase reporter assays revealed that depletion of NEAT1 in 293 T cells inhibited the luciferase activity of ATGL-WT but not ATGLMUT. Further, inhibition of miR-124-3p reversed this decrease in luciferase activity for ATGL-WT, but not for ATGL-MUT. Data are expressed as mean \pm SD. Statistical significance was concluded at ${ }^{* *} P<0.01$. NS represents no statistical significance. (TIF $685 \mathrm{~kb}$ )

Additional file 13: Figure S11. The effect of miR-124-3p on lipolysis. A. Treatment with miR-124-3p mimic decrease intracellular DAG levels in SKhep-1 and HCCLM3 cells B. Treatment with miR-124-3p mimic decrease intracellular FFA levels in SK-hep-1 and HCCLM3 cells. Data are expressed as mean \pm SD of three independent experiments. Statistical significance was concluded at ${ }^{*} P<0.01,{ }^{* *} P<0.001$. (TIF $832 \mathrm{~kb}$ )

Additional file 14: Figure S12. NEAT1 and miR-124-3p mRNA was aberrantly expressed in 5 pairs of HCC and matched non-tumor tissues. A. Real-time PCR analysis of NEAT1 and miR-124-3p expression in five pairs of HCC and matched non-tumor tissues. Data are expressed as mean \pm SD of three independent experiments. (TIF $678 \mathrm{~kb}$ )

\section{Abbreviations}

ATGL/PNPLA2: Adipose triglyceride lipase; DAG: Diacylglycerol; FAS: Fatty acid synthesis; FFA: Free fatty acid; HCC: Hepatocellular carcinoma; HSL: Hormone-sensitive lipase; IHC: Immunohistochemistry; MAG: Monoacylglycerol; MAGL: Monoglyceride lipase; NEAT1: Nuclear paraspeckle assembly transcript 1; PCR: Polymerase chain reaction; PPARa: Peroxisome proliferator activated receptor alpha; TAG: Triglyceride

\section{Funding}

This study was supported by National Key Program for Science and Technology Research and Development (2016YFC0106500, 2016YFC0106503, 2016YFC0905902), Changjiang Scholars and Innovative Research Team in
University (grant no.: IRT1122), National Natural Science Foundation of China (grant nos.: 81602058), University Nursing Program for Young Scholars with Creative Talents in Heilongjiang Province, (UNPYSCT-2016200), Harbin Medical University Scientific Research Innovation Fund, (2017LCZX20), The First Affiliated Hospital of Harbin Medical University Scientific Research Innovation Fund, (2017 L005), Program for National Natural Science Foundation of China (81301807), General Financial Grant from the China Postdoctoral Science Foundation (2016 M590292), Heilongjiang Postdoctoral Fund (LBH-Z16100), Heilongjiang Nature Science Foundation (LC2013C31), Wu Liande Science Foundation for Young Scholars of Harbin Medical University (WLD-QN1403). The funder had no role in study design, data collection and analysis, decision to publish, or preparation of the manuscript.

\section{Availability of data and materials}

All data generated or analysed during this study are included in this published article [and its supplementary information files].

\section{Authors' contributions}

LXL designed this research. XRL, YJL, RPS and GCY contribute equally to this work. XRL, YJL, RPS and GCY performed most experiments in this work and XRL drafted this manuscript. JHH, YLL and SHP helped with the western bolt experiments. MXZ, YL, YW and FZM helped with the CCK-8 and colony formation assay. YZL and TSZ helped with PCR experiments. YFC, JBW, BZ, and XS helped with the animal experiment. All authors read and approved the final manuscript.

\section{Ethics approval and consent to participate}

HCC tissue and paired normal adjacent tissue samples were acquired from patients undergoing a surgical procedure at the First Affiliated Hospital of Harbin Medical University (Harbin, China).Patient consent was obtained prior to the initiation of the study.

\section{Consent for publication}

We have obtained consents to publish this paper from all the participants of this study.

\section{Competing interests}

The authors declare that they have no competing interests.

\section{Publisher's Note}

Springer Nature remains neutral with regard to jurisdictional claims in published maps and institutional affiliations.

\section{Author details}

'Department of Hepatic Surgery, The First Affiliated Hospital of Harbin Medical University, Key Laboratory of Hepatosplenic Surgery, Ministry of Education, Harbin, Heilongjiang Province, China. ${ }^{2}$ Key Laboratory of Hepatosplenic Surgery, Ministry of Education, The First Affiliated Hospital of Harbin Medical University, Harbin, Heilongjiang Province, China. ${ }^{3}$ Department of Gastrointestinal Medical Oncology, The Affiliated Tumour Hospital of Harbin Medical University, Harbin, Heilongjiang, China. ${ }^{4}$ Department of Pharmacology (the State-Province Key Laboratories of Biomedicine-Pharmaceutics of China, Key Laboratory of Cardiovascular Research, Ministry of Education), Harbin Medical University, Harbin, China.

Received: 20 August 2017 Accepted: 25 April 2018

Published online: 15 May 2018

\section{References}

1. Jemal A, Bray F, Center MM, Ferlay J, Ward E, Forman D. Global cancer statistics. CA Cancer J Clin. 2011;61:69-90.

2. Tanaka S, Arii S. Molecular targeted therapies in hepatocellular carcinoma. Semin Oncol. 2012;39:486-92.

3. Menendez JA, Lupu R. Fatty acid synthase and the lipogenic phenotype in cancer pathogenesis. Nat Rev Cancer. 2007;7:763-77.

4. Zaidi N, Lupien L, Kuemmerle NB, Kinlaw WB, Swinnen JV, Smans K. Lipogenesis and lipolysis: the pathways exploited by the cancer cells to acquire fatty acids. Prog Lipid Res. 2013;52:585-9.

5. Bolsoni-Lopes A, Alonso-Vale MI. Lipolysis and lipases in white adipose tissue - an update. Arch Endocrinol Metab. 2015;59:335-42. 
6. Nomura DK, Long JZ, Niessen S, Hoover HS, Ng SW, Cravatt BF. Monoacylglycerol lipase regulates a fatty acid network that promotes cancer pathogenesis. Cell. 2010;140:49-61.

7. Rajasekaran D, Jariwala N, Mendoza RG, Robertson CL, Akiel MA, Dozmorov M, Fisher PB, Sarkar D. Staphylococcal nuclease and tudor domain containing 1 (SND1 Protein) promotes hepatocarcinogenesis by inhibiting Monoglyceride Lipase (MGLL). J Biol Chem. 2016;291:10736-46.

8. Zagani R, El-Assaad W, Gamache I, Teodoro JG. Inhibition of adipose triglyceride lipase (ATGL) by the putative tumor suppressor GOS2 or a small molecule inhibitor attenuates the growth of cancer cells. Oncotarget. 2015; 6:28282-95.

9. Souquere S, Beauclair G, Harper F, Fox A, Pierron G. Highly ordered spatial organization of the structural long noncoding NEAT1 RNAs within paraspeckle nuclear bodies. Mol Biol Cell. 2010;21:4020-7.

10. Yang C, Li Z, Li Y, X X R, Wang Y, Tian Y, Chen W. Long non-coding RNA NEAT1 overexpression is associated with poor prognosis in cancer patients: a systematic review and meta-analysis. Oncotarget. 2017;8:2672-80.

11. Zhen L, Yun-Hui L, Hong-Yu D, Jun M, Yi-Long Y. Long noncoding RNA NEAT1 promotes glioma pathogenesis by regulating miR-449b-5p/c-Met axis. Tumour Biol. 2016;37:673-83.

12. Cao J, Zhang Y, Yang J, He S, Li M, Yan S, Chen Y, Qu C, Xu L. NEAT1 regulates pancreatic cancer cell growth, invasion and migration though mircroRNA-335-5p/c-met axis. Am J Cancer Res. 2016;6:2361-74.

13. Fujimoto A, Furuta M, Totoki Y, Tsunoda T, Kato M, Shiraishi Y, Tanaka H, Taniguchi H, Kawakami Y, Ueno M, et al. Whole-genome mutational landscape and characterization of noncoding and structural mutations in liver cancer. Nat Genet. 2016;48:500-9.

14. Yang $G$, Liang $Y$, Zheng T, Song R, Wang J, Shi H, Sun B, Xie C, Li Y, Han J, et al. FCN2 inhibits epithelial-mesenchymal transition-induced metastasis of hepatocellular carcinoma via TGF-beta/Smad signaling. Cancer Lett. 2016; 378:80-6.

15. Chen C, Ridzon DA, Broomer AJ, Zhou Z, Lee DH, Nguyen JT, Barbisin M, Xu $\mathrm{NL}$, Mahuvakar VR, Andersen MR, et al. Real-time quantification of microRNAs by stem-loop RT-PCR. Nucleic Acids Res. 2005;33:e179.

16. Wang J, Xie C, Pan S, Liang Y, Han J, Lan Y, Sun J, Li K, Sun B, Yang G, et al. $\mathrm{N}$-myc downstream-regulated gene 2 inhibits human cholangiocarcinoma progression and is regulated by leukemia inhibitory factor/MicroRNA-181C negative feedback pathway. Hepatology. 2016;64:1606-22.

17. Chakrabarti P, Kandror KV. Adipose triglyceride lipase: a new target in the regulation of lipolysis by insulin. Curr Diabetes Rev. 2011;7:270-7.

18. Li P, Ruan X, Yang L, Kiesewetter K, Zhao Y, Luo H, Chen Y, Gucek M, Zhu J, Cao H. A liver-enriched long non-coding RNA, IncLSTR, regulates systemic lipid metabolism in mice. Cell Metab. 2015;21:455-67.

19. Zhao Z, Bai J, Wu A, Wang Y, Zhang J, Wang Z, Li Y, Xu J, Li X. Co-LncRNA: investigating the IncRNA combinatorial effects in $\mathrm{GO}$ annotations and KEGG pathways based on human RNA-Seq data. Database (Oxford). 2015. https:// doi.org/10.1093/database/bav082

20. Yuan SX, Wang J, Yang F, Tao QF, Zhang J, Wang LL, Yang Y, Liu H, Wang ZG, Xu QG, et al. Long noncoding RNA DANCR increases stemness features of hepatocellular carcinoma by derepression of CTNNB1. Hepatology. 2016; 63:499-511.

21. Adriaens C, Standaert L, Barra J, Latil M, Verfaillie A, Kalev P, Boeckx B, Wijnhoven PW, Radaelli E, Vermi W, et al. p53 induces formation of NEAT1 IncRNA-containing paraspeckles that modulate replication stress response and chemosensitivity. Nat Med. 2016;22:861-8.

22. Blume CJ, Hotz-Wagenblatt A, Hullein J, Sellner L, Jethwa A, Stolz T, Slabicki M, Lee K, Sharathchandra A, Benner A, et al. p53-dependent non-coding RNA networks in chronic lymphocytic leukemia. Leukemia. 2015;29:2015-23.

23. Guil S, Esteller M. RNA-RNA interactions in gene regulation: the coding and noncoding players. Trends Biochem Sci. 2015;40:248-56.

24. Lv J, Fan HX, Zhao XP, Lv P, Fan JY, Zhang Y, Liu M, Tang H. Long noncoding RNA Unigene56159 promotes epithelial-mesenchymal transition by acting as a ceRNA of miR-140-5p in hepatocellular carcinoma cells. Cancer Lett. 2016:382:166-75.

25. Liang $H$, Zhang J, Zen $K$, Zhang $C Y$, Chen $X$. Nuclear microRNAs and their unconventional role in regulating non-coding RNAs. Protein Cell. 2013;4: 325-30.

26. Leucci E, Patella F, Waage J, Holmstrom K, Lindow M, Porse B, Kauppinen S, Lund AH. microRNA-9 targets the long non-coding RNA MALAT1 for degradation in the nucleus. Sci Rep. 2013;3:2535.
27. Jeon SM, Chandel NS, Hay N. AMPK regulates NADPH homeostasis to promote tumour cell survival during energy stress. Nature. 2012;485:661-5.

28. Ong KT, Mashek MT, Davidson NO, Mashek DG. Hepatic ATGL mediates PPAR-alpha signaling and fatty acid channeling through an L-FABP independent mechanism. J Lipid Res. 2014;55:808-15.

29. Georgiadi A, Kersten $\mathrm{S}$. Mechanisms of gene regulation by fatty acids. Adv Nutr. 2012;3:127-34

30. Shah YM, Morimura K, Yang Q, Tanabe T, Takagi M, Gonzalez FJ. Peroxisome proliferator-activated receptor alpha regulates a microRNA-mediated signaling cascade responsible for hepatocellular proliferation. Mol Cell Biol. 2007;27:4238-47.

31. Shariat SF, Chade DC, Karakiewicz PI, Ashfaq R, Isbarn H, Fradet Y, Bastian PJ, Nielsen ME, Capitanio U, Jeldres C, et al. Combination of multiple molecular markers can improve prognostication in patients with locally advanced and lymph node positive bladder cancer. J Urol. 2010;183:68-75.

32. Sallam T, Jones MC, Gilliland T, Zhang L, Wu X, Eskin A, Sandhu J, Casero D, Vallim TQ, Hong C, et al. Feedback modulation of cholesterol metabolism by the lipid-responsive non-coding RNA LeXis. Nature. 2016;534:124-8.

33. Cooper DR, Carter G, Li P, Patel R, Watson JE, Patel NA. Long non-coding RNA NEAT1 associates with SRp40 to temporally regulate PPARgamma2 splicing during adipogenesis in 3T3-L1 cells. Genes (Basel). 2014;5:1050-63.

34. Lamming DW, Sabatini DM. A central role for mTOR in lipid homeostasis. Cell Metab. 2013;18:465-9.

35. Wojcik M, Mac-Marcjanek K, Wozniak LA. Physiological and pathophysiological functions of SIRT1. Mini Rev Med Chem. 2009;9:386-94.

36. Chen X, Xun K, Chen L, Wang Y. TNF-alpha, a potent lipid metabolism regulator. Cell Biochem Funct. 2009;27:407-16.

37. de la Rosa Rodriguez MA, Kersten S. Regulation of lipid droplet-associated proteins by peroxisome proliferator-activated receptors. Biochim Biophys Acta. 2017;1862:1212-20.

38. Choudhry H, Albukhari A, Morotti M, Haider S, Moralli D, Smythies J, Schodel J, Green CM, Camps C, Buffa F, et al. Tumor hypoxia induces nuclear paraspeckle formation through HIF-2alpha dependent transcriptional activation of NEAT1 leading to cancer cell survival. Oncogene. 2015;34: $4482-90$.

39. Ke H, Zhao L, Feng $X$, Xu H, Zou L, Yang Q, Su X, Peng L, Jiao B. NEAT1 is required for survival of breast cancer cells through FUS and miR-548. Gene Regul Syst Bio. 2016;10:11-7.

40. Chakravarty D, Sboner A, Nair SS, Giannopoulou E, Li R, Hennig S, Mosquera JM, Pauwels J, Park K, Kossai M, et al. The oestrogen receptor alpharegulated IncRNA NEAT1 is a critical modulator of prostate cancer. Nat Commun. 2014:5:5383.

41. Jen J, Tang YA, Lu YH, Lin CC, Lai WW, Wang YC. Oct4 transcriptionally regulates the expression of long non-coding RNAs NEAT1 and MALAT1 to promote lung cancer progression. Mol Cancer. 2017;16:104

42. Zeng C, Xu Y, Xu L, Yu X, Cheng J, Yang L, Chen S, Li Y. Inhibition of long non-coding RNA NEAT1 impairs myeloid differentiation in acute promyelocytic leukemia cells. BMC Cancer. 2014:14:693.

43. Mello SS, Sinow C, Raj N, Mazur PK, Bieging-Rolett K, Broz DK, Imam JFC, Vogel H, Wood LD, Sage J, et al. Neat1 is a p53-inducible lincRNA essential for transformation suppression. Genes Dev. 2017;31:1095-108.

44. Idogawa M, Ohashi T, Sasaki Y, Nakase H, Tokino T. Long non-coding RNA NEAT1 is a transcriptional target of p53 and modulates p53-induced transactivation and tumor-suppressor function. Int J Cancer. 2017;140:2785-91.

45. Zhang J, Li Y, Dong M, Wu D. Long non-coding RNA NEAT1 regulates E2F3 expression by competitively binding to miR-377 in non-small cell lung cancer. Oncol Lett. 2017;14:4983-8.

46. Jiang X, Zhou Y, Sun AJ, Xue JL. NEAT1 contributes to breast cancer progression through modulating miR-448 and ZEB1. J Cell Physiol. 2018. https://doi.org/10.1002/jcp.26470

47. Peng $W$, Wang Z, Fan H. LncRNA NEAT1 impacts cell proliferation and apoptosis of colorectal cancer via regulation of Akt signaling. Pathol Oncol Res. 2017;23:651-6.

48. Khan SA, Sathyanarayan A, Mashek MT, Ong KT, Wollaston-Hayden EE, Mashek DG. ATGL-catalyzed lipolysis regulates SIRT1 to control PGC-1alpha/ PPAR-alpha signaling. Diabetes. 2015;64:418-26.

49. Desvergne B, Michalik L, Wahli W. Transcriptional regulation of metabolism. Physiol Rev. 2006;86:465-514. 